ACCEPTED MANUSCRIPT

\title{
The plane- and spherical- wave descriptions of electromagnetic radiation: A comparison and discussion of their relative merits
}

To cite this article before publication: Garth Andrew Jones et al 2018 Eur. J. Phys. in press https://doi.org/10.1088/1361-6404/aac366

\section{Manuscript version: Accepted Manuscript}

Accepted Manuscript is "the version of the article accepted for publication including all changes made as a result of the peer review process, and which may also include the addition to the article by IOP Publishing of a header, an article ID, a cover sheet and/or an 'Accepted Manuscript' watermark, but excluding any other editing, typesetting or other changes made by IOP Publishing and/or its licensors"

This Accepted Manuscript is (C) 2018 European Physical Society.

During the embargo period (the 12 month period from the publication of the Version of Record of this article), the Accepted Manuscript is fully protected by copyright and cannot be reused or reposted elsewhere.

As the Version of Record of this article is going to be / has been published on a subscription basis, this Accepted Manuscript is available for reuse under a CC BY-NC-ND 3.0 licence after the 12 month embargo period.

After the embargo period, everyone is permitted to use copy and redistribute this article for non-commercial purposes only, provided that they adhere to all the terms of the licence https://creativecommons.org/licences/by-nc-nd/3.0

Although reasonable endeavours have been taken to obtain all necessary permissions from third parties to include their copyrighted content within this article, their full citation and copyright line may not be present in this Accepted Manuscript version. Before using any content from this article, please refer to the Version of Record on IOPscience once published for full citation and copyright details, as permissions will likely be required. All third party content is fully copyright protected, unless specifically stated otherwise in the figure caption in the Version of Record.

View the article online for updates and enhancements. 


\title{
The Plane- and Spherical- Wave Descriptions of Electromagnetic
} Radiation: A Comparison and Discussion of Their Relative Merits

\author{
G. A. Jones ${ }^{1}$ and R. Grinter ${ }^{1}$ \\ ${ }^{1}$ School of Chemistry, University of East Anglia, Norwich Research Park, Norwich NR4
} 7TJ, United Kingdom.

garth,jones@uea.ac.uk

r.grinter@uea.ac.uk

\section{Abstract}

In this review article we compare two different mathematical descriptions of electromagnetic radiation. Namely one describing the fields in terms of plane-waves, and the other using spherical-waves. We analyse the different approaches in terms of the multipole expansion, and weigh-up advantages and disadvantages of the two approaches. The focus is within the context of a non-relativistic quantum electrodynamical formulation, and we consider processes related to light-matter interactions, such as electronic energy transfer. We also include an analysis of symmetry and group theoretical considerations. Significant numerical details are provided in the appendices that may be useful to readers who want to apply the theory to applications.

\section{Introduction}

The manipulation of electromagnetic radiation (EMR) at the level of photons is a highly active field of research. A complete understanding of photons at the quantum mechanical level is essential to the development of new technologies such as those centred around structured light [1], near-field imaging techniques [2-4] and ghost imaging [5, 6]. It will very likely also be key to many emerging technologies such as those associated with quantum computers $[7,8]$ and quantum communication $[9,10]$. Key to this understanding is being able to describe the photonic fields mathematically. In some applications a preferred basis may be more desirable. In this review, we weigh-up the merits of two of the most common bases for describing electromagnetic radiation, namely the plane (PW) and spherical wave (SW) descriptions of photonic fields.

Early attempts to unite quantum theory with Maxwell's equations, to produce an integrated and self-consistent description of EMR and its interaction with atoms and molecules, in which all elements of the theory are quantised were made rapidly; notably by Dirac [11, 12] and a little later by Jordan and Pauli [13]. Extraordinary progress in the 
second half of the $20^{\text {th }}$ century led to the sophisticated theory we now know as quantum electrodynamics (QED) [14-16]. Upon looking a little closer at these developments, a somewhat surprising detail emerges. Some of the earliest theoretical papers focused upon a description of EMR in terms of vector spherical harmonics (VSHs) and Bessel functions (BFs) - referred to in this review as the spherical wave approach. [17, 18] This description was elaborated by Franz, [19] and pursued especially by nuclear physicists, presumably because it is strongly angular momentum focused. The approach permits ready use of powerful mathematical concepts based upon angular momentum theory; vector coupling through Clebsch-Gordan coefficients or Wigner symbols, irreducible spherical tensor algebra and multipole fields [20-21]. It is also readily extendable to situations in which nuclear transitions are accompanied by large changes of angular momentum, [22], i.e. when $\Delta J \geq 2$. Though widely applied in nuclear physics, this type of description appears to have been little used in atomic and molecular physics, or in the analysis of the many complex quantum optical phenomena, such as structured light applications including LaguerreGaussian beams, which have been so extensively studied in recent years [23, 24]. In these areas of research a plane wave description, implemented through Cartesian tensor algebra, is the method of choice. Few, if any, attempts have been made to compare these two approaches, namely the spherical and plane wave description of photonic fields, to the same problems. However, these descriptions are complementary and some cross-fertilization could be valuable; especially for those working in quantum optics and in particular those with a focus on structured light applications and in the area of quantum information.

Accordingly, in this review we compare the PW and SW methods, to illustrate their strengths and weaknesses, and to show how these different formulations can be related. In so doing we shall make use of Cartesian $(\vec{x}, \vec{y}, \hat{\vec{z}})$ and spherical polar $(\vec{R}, \vec{\Theta}, \vec{\Phi})$ unit vectors, which may be source- or photon-based. It is important to note that the relationships of source-based to photon-based vectors depends upon the direction of the wave vector, $\hat{\vec{k}}$; i.e. they differ for in-coming and out-going photons. In the case of the spherical description of photonic fields, the radial component makes use of Bessel, Neumann and Hankel functions of half-integral order [25, 26]. EM waves described by Bessel functions, $j_{l}(r)$, and by Neumann functions, $n_{l}(r)$, are stationary waves for all values of $r$, including infinity, whilst their combination in the form of the Hankel functions, $h_{l}^{(1)}(r)$ and $h_{l}^{(2)}(r)$, give rise running waves for all $r$ [27]. The direction in which the photon is travelling depends upon the choice of sign in the time-dependence factor. Combinations of $\exp (-\mathrm{i} \omega t)$ with $h_{l}{ }^{(1)}(r)$ and $h_{l}{ }^{(2)}(r)$ 
describe out-going and in-coming photons respectively, and we shall adopt this timedependence throughout this review. If $\exp (+\mathrm{i} \omega t)$ is chosen the situation is reversed.

In this work we assume a non-relativistic quantum electrodynamical formulation within the Coulomb gauge. This is because the electrons of interest are bound within the valence shells of atoms or molecules. Although we won't be discussing relativistic applications typically written in the Lorentz-covariant 4-vector formulation in this review, the ideas presented here are general and transferrable to such applications.

\section{Background Theory}

\subsection{The quantum mechanical nature of the photon.}

It is informative to briefly review the quantum mechanical description of the photon. There are a number of good references to which the reader is pointed for more detailed information [16, 28-31]. In regions free of charges and currents, Maxwell's equations for the EMR field can be transformed into a differential equation having the same form for the vector potential, $\vec{A}$, the electric field, $\vec{E}$, and the magnetic field, $\vec{B}$; namely:

$\nabla^{2} \vec{X}-\frac{1}{c^{2}} \frac{\partial^{2} \vec{X}}{\partial t^{2}}=0$

where $\vec{X}=\vec{A}, \vec{E}$ or $\vec{B}$. This is a wave equation for which there are many solutions, mostly in terms of sine, cosine and exponential functions or combinations of them. For a frequency $v$ the energy of the wave is given by Planck's relationship:

$E=h v=\hbar \omega$

where $\omega$ is the angular frequency, $2 \pi v$. The field is envisaged as resulting from the motions of a system of harmonic oscillators and, since in free space there is no restriction on their frequencies, there is no quantisation and any energy is possible. The usual means by which quantisation is introduced is to restrict the possible values of $v$ by enclosing the oscillators within a "box" or requiring that the waves be subject to periodic boundary conditions. Either form of restriction means that only certain wavelengths, $\lambda$, are possible and hence only the corresponding frequencies and energies. In the case of a cubic box of side $L$, for example, the only wavelengths (modes) allowed are those for which

$k_{x}=\frac{2 \pi n_{x}}{L}, \quad k_{y}=\frac{2 \pi n_{y}}{L}$ and $\quad k_{z}=\frac{2 \pi n_{z}}{L}$

where $k=2 \pi / \lambda$ is the wave number and the $n_{i}$ are integers. The solutions of the wave equation for $\vec{A}, \vec{E}$ or $\vec{B}$ may be written as a Fourier series in the allowed modes. An example of such a mode expansion for the vector potential is: 


$$
\vec{A}(r, t)=\sum_{k}\left\{a_{k}(t) \exp (\mathrm{i} \vec{k} \cdot \vec{r})+a_{k}^{*}(t) \exp (-\mathrm{i} \vec{k} \cdot \vec{r})\right\}
$$

Where the $a_{k}(t)$ are time-dependent amplitude coefficients and the second term in parentheses is the complex conjugate of the first.

The excitations of these modes represent the photons, and since these are bosons there is no limit on the number which can occupy any particular mode. A single mode is symbolized by the ket $|n(\vec{k}, \sigma)\rangle$ where $n$ is the number of photons in the mode, $\vec{k}$ the wave vector and $\sigma$ the photon's polarization. The complete radiation field is expressed as a product of the individual modes as:

$$
\Psi=\left|n_{1}\left(\vec{k}_{1}, \sigma_{1}\right), n_{2}\left(\vec{k}_{2}, \sigma_{2}\right), n_{3}\left(\vec{k}_{3}, \sigma_{3}\right) \ldots\right\rangle \text {. }
$$

The Hamiltonian operator and its application to determine the eigen-energies of a harmonic oscillator is described in numerous elementary books on quantum mechánics. Following the procedure of second quantisation, the Hamiltonian can be written as,

$$
\hat{H}=\left(\hat{a}^{\dagger} \hat{a}+1 / 2\right) \hbar \omega \text {. }
$$

The operators $\hat{a}^{\dagger}$ and $\hat{a}$ have the following remarkable properties:

$$
\hat{a}|n(\vec{k}, \sigma)\rangle=n^{1 / 2}|(n-1)(\vec{k}, \sigma)\rangle
$$

and

$$
\hat{a}^{\dagger}|n(\vec{k}, \sigma)\rangle=(n+1)^{1 / 2}|(n+1)(\vec{k}, \sigma)\rangle,
$$

from which we see that $\hat{a}$ reduces the number of photons in the mode by one while $\hat{a}^{\dagger}$ increases it by one. For this reason they are called the annihilation and creation operators respectively, and they have exactly the effect needed to describe the interaction of EMR with atoms and molecules, when it is accompanied by the absorption or emission of a photon.

Though we have now found a way to quantise the oscillator energy levels and express the electromagnetic field in terms of photons, Eq. (4) is a classical expression which requires further development. We may recall the procedure in basic quantum mechanics where the canonical variables of a classical expression are "promoted" to operators to generate a quantum-mechanical counterpart. One of the most important examples being the replacement of linear momentum along $x$ by a differential operator; symbolically:

$$
m v_{x}=p_{x} \Rightarrow-\mathrm{i} \hbar \partial / \partial x
$$

Similarly, in passing from classical electrodynamics to quantum electrodynamics, the field amplitudes, $a_{k}(t)$, of the classical equations are replaced by the above creation and annihilation operators, which describe discrete excitations and de-excitations in the photonic 
field. Photons are therefore regarded as elementary excitations of an optical mode, with a specific angular frequency and polarization.

When classical field amplitudes are replaced by creation and annihilation operators Eq. (4) becomes:

$$
\begin{aligned}
& \vec{A}(r, t)=\sum_{k, \sigma}\left(\frac{\hbar}{2 \varepsilon_{0} c k V}\right)^{1 / 2} \times \\
& \left\{e^{\sigma}(k) a_{k}^{\sigma}(k) \exp [\mathrm{i}(\vec{k} \cdot \vec{r}-\omega t)]+e^{* \sigma}(k)(k) a_{k}^{\dagger \sigma}(k) \exp [-\mathrm{i}(\vec{k} \cdot \vec{r}-\omega t)]\right\} .
\end{aligned}
$$

Here $\varepsilon_{0}$ is the permittivity of free space, $V$ the quantisation volume and $e^{\sigma}$ the photon polarization vector.

\subsection{The interaction of EMR with matter: the multipolar Hamiltonian}

The focus of quantum electrodynamics is the interaction of EMR with matter, and unlike semi-classical theories, both radiation and matter are treated in a fully quantum mechanical way, and can be developed in terms of both Lagrangian and Hamiltonian formulations. The interaction Hamiltonians are of particular interest are because they dictate the nature of the emission and absorption. When atoms and molecules form sources for currents, the multipolar expansion is employed. That is to say, in matter electric and magnetic polarization results from/charge and current densities. Charge densities have contributions from free and bound charges, while current densities originate from electric polarization and magnetic currents generated by motion of the bound charges. Convective and Röntgen currents also make contributions in certain situations. Decomposition of matter in this way leads to the multipolar form of the theory, as outlined below.

This work is focused towards atomic and molecular structure, however it should be noted that much of the theory is also applicable to nuclei. In the molecular case it should be noted that we consider only the electronic degrees of freedom and hence any vibrational contributions are assumed completely decoupled from the light-matter interaction. The Power-Zienau-Woolley (PZW) Hamiltonian in the exact multipolar form is given by,

$$
\hat{H}_{m u l t}=\sum_{\xi} \hat{H}_{m o l}(\xi)+\hat{H}_{\text {rad }}+\hat{H}_{\text {int }}(\xi)
$$

where the terms on the right correspond to the Hamiltonians for the molecule the radiation and their interaction respectively. If more than one molecule or atom is present they are each coupled to the radiation, but not to each other; all intermolecular interaction is via the field. The introduction of coupling between the radiation field and a molecule (or any other 
interacting species) corresponds to the substitution (12) which is known as the principle of minimal electromagnetic coupling.

$\overrightarrow{\hat{p}} \Rightarrow \overrightarrow{\hat{p}}-e \vec{A}(q)$

A specific example is the interaction between EMR and the single electron of the hydrogen atom resulting in the absorption (annihilation) of the photon and the excitation of the atom, which takes the form.

$$
\hat{\mathrm{H}}_{\mathrm{int}}=\frac{e}{m} \overrightarrow{\hat{p}} \cdot \vec{A}(r)
$$

Equation 13, as it stands, is not particularly informative. Rather more insight may be obtained from the expression for the multipolar Hamiltonian which, as outlined in reference [6], is expressible as a series,

$$
\hat{\mathrm{H}}_{\mathrm{int}}(\xi)=\varepsilon_{0}^{-1} \mu(\xi) \cdot d^{\perp}\left(\vec{R}_{\xi}\right)+\varepsilon_{0}^{-1} Q(\xi): \nabla d^{\perp}\left(\vec{R}_{\xi}\right)-\varepsilon_{0}^{-1} \Omega(\xi) \vdots \nabla \nabla d^{\perp}\left(\vec{R}_{\xi}\right)-\ldots
$$

Where $\mu(\xi), Q(\xi)$ and $\Omega(\xi)$ represent the electric dipole, electric quadrupole and the electric octupole components of the interacting species, $\xi$, and $d^{\perp}\left(\vec{R}_{\xi}\right)$ is the transverse field displacement operator associated with the photon. In Eq. (14) we see how the radiation interacts with the various multipole moments of $\xi$. Explicit calculations of these higher order terms typically involve the use of tensor algebra, where an increase in the order of the $2^{l}$ pole corresponds mathematically to an increase in the rank of the tensor (i.e. the dipole term requires evaluating a first order tensor, the quadrupole a second rank tensor, and so on). When describing electronic transitions, within the context of the multipolar formulation of QED, the emission and excitation processes, are typically described in terms of the leading order of the transition. For example, a transition that is dipole allowed is labelled E1, while a transition that is dipole forbidden, but quadrupole allowed is labelled E2. There are equivalent magnetic transitions labelled M1, M2, etc [32].

\subsection{Helmholtz decomposition of (photonic) fields.}

Central to the theory of quantum electrodynamics is that all interactions between electrons occur via the exchange of virtual photons. In the case of non-relativistic QED, where the electrons in question are typically bound to the valence orbitals of atoms and molecules, the Coulomb gauge is employed. Calculations, such as that of the electronic coupling between two molecules, are carried out formally, by summing over all possible virtual photon modes. In many applications it is informative to consider how the character of a virtual photon changes as we move from the near- to the far-zone. The implications of 
whether a photonic event occurs in the near- intermediate- or far-zone could have could influence design principles of particular metamaterials [33, 34].

The regimes of the interaction between electronic species are defined in terms of the distance between the species $R$ and the wavevector of the mediating virtual photon, $k$. Broadly speaking, near-zone events occur when $k R \ll 1$, intermediate-zone when $k R \sim 1$ and far-zone events occur when $k R \gg 1$. In real terms, this means that interactions between molecules that are less than several nanometers apart are near-zone events, while experiments that involve traditional photon detectors occur well above the far-zone limit. An increasingly important regime is the intermediate-zone, which occurs at around 10 - 100 nanometers for optical processes. This is of course the approximate dimension of many metamaterials. In these cases, both near- and far-zone considerations should be taken into account.

The Helmholtz decomposition theorem states that any vector field can be separated in zero-curl (irrotational) and zero-divergence (solenoidal) components,

$$
\begin{gathered}
\vec{F}(\vec{r})=\vec{F}^{\operatorname{Sol}}(\vec{r})+\vec{F}^{I r r}(\vec{r}) \\
\nabla \cdot \vec{F}^{\text {Sol }}=0 \\
\nabla \times \vec{F}^{I r r}=0
\end{gathered}
$$

Within the context of Coulomb gauge QED, which typically employs the PZW Hamiltonian [35-37], irrotational parts of the EM fields disappear and the remaining transverse fields are defined as being orthogonal to $\vec{k}$ (and specifically not with respect to the spatial coordinate $\vec{r})$. Nevertheless, at the quantum mechanical level, the description of the field in terms of its components is quite different in the near-zone compared to the far-zone. In the near-zone, the emitted virtual photon is still close to the source, and hence because of the high degree of certainty in the photon's position, Heisenberg's Principle dictates that there is an associated high degree of uncertainty in the momentum; including its direction. This means that in summing over all modes of the virtual photons, as well as transverse fields, there will be associated longitudinal fields, with respect to the displacement vector $\vec{r}$. As the photon moves away from the source, entering the far-zone, its momentum becomes well defined 
and the longitudinal components disappear, leaving only transverse components of the electric and magnetic fields.

Consequently, when considering near-field photonic interactions, it is important tơ think about longitudinal as well as transverse components of the EM fields. [38-40] As will be shown in subsequent sections of this review, the spherical wave description of the mediating photons allows one to decompose the fields in transverse and longitudinal components naturally, whereas in the case of the plane-wave description a judicious choice of Cartesian coordinates is usually required.

\subsection{The plane-wave (PW) description of light}

In the plane wave description, the solution of Eq. (1) is:

$$
\vec{X}=\vec{X}_{0} \exp \mathrm{i}[\vec{k} \cdot \vec{r}-\omega t]
$$

where $\vec{X}_{0}$ is a constant amplitude factor, $\omega$ the angular frequency and $\vec{k}$ the wave vector. In the PW description of light, neglecting time-dependence, the exponential function is combined with a polar unit vector, $\hat{\vec{e}}_{1 n}$, to represent the field direction and the exponential is expanded in the well-known manner, as shown in Eq. (19):

$$
\hat{\vec{e}}_{1 n} \exp [\mathrm{i} \vec{k} \cdot \vec{r}]=\hat{\vec{e}}_{1 n}\left[1+\mathrm{i} \vec{k} \cdot \vec{r}+\frac{(\mathrm{i} \vec{k} \cdot \vec{r})^{2}}{2 !}+\frac{(\mathrm{i} \vec{k} \cdot \vec{r})^{3}}{3 !}+\cdots\right]
$$

Here the subscript $n$ represents the direction of polarization, and the 1 indicates that this vector has three $(n= \pm 1,0)$ components. This expression for the expansion can be exploited either analytically or numerically, and utilized for calculational purposes, within the context of classical or quantum electrodynamics. As detailed below, the expression (19) can be used to identify multipole terms directly, and appropriate truncation of the series will depend on the particular application.

\subsection{The spherical-wave (SW) description of light}

In the SW description, again neglecting time-dependence, the exponential function is usually, though not invariably, combined with an axial unit vector, $\hat{\vec{e}}_{1 n}$, to represent the 
field direction and, for radiation propagating in a general direction characterised by the polar angles $\alpha$ and $\beta$. The exponential is expanded according to Eq. (20) [41]:

$$
\begin{gathered}
\hat{\vec{e}}_{1 n} \exp [\mathrm{i} \vec{k} \cdot \vec{r}]=4 \pi \sum_{J} \sum_{l} \sum_{M} \mathrm{i}^{l} j_{l}(k r) \mid l, m(\alpha, \beta)> \\
<l 1 m n|J M>| J, l, M(\vartheta, \varphi)>
\end{gathered}
$$

Here $j_{l}(k r)$ is a spherical Bessel function, $\mid l, m(\alpha, \beta)>$ a normalised scalar spherical harmonic (SSH), $\mid J, l, M(\vartheta, \varphi)>$ a normalized VSH $[42,43]$ and $\langle l 1 m n| J M>$ a Clebsch-Gordan coefficient or Wigner $3 j$ symbol $[27,41]$. For the description of the field surrounding an electric or magnetic multipole one obtains, depending upon the detailed structure of the multipole, expressions in which the angular VSH's are combined with radial Hankel functions, $h_{l}^{(1)}(r)$ and $h_{l}^{(2)}(r)$ which, in combination with a time factor of $\exp (-\mathrm{i} \omega t)$, describe out-going and in-coming waves respectively [44].

With regard to the use of expansion Eq. (20), the following general points may be made: (1) Its apparent complexity is largely an illusion since, in any particular application, only one value of $M$ and one or two values of $J$ are involved, and $l$ can never be other than $J$ or $J \pm 1$. (2) The integer values of the parameters of the $3 j$ symbol which result in a nonvanishing coefficient are strictly limited by the laws governing the addition of angular momenta, and values have been extensively tabulated, e.g. [45, 46].

That said, it is clear that Eq. (20) is directly applicable only to spherically symmetrical situations, which accounts for its predominant use in nuclear physics. Where it can be applied, it gives angular intensity distribution and retarded radial functions automatically [44]. Furthermore, when the molecular systems possess some degree of symmetry, the connection between the VSHs and the irreducible representations (IRs) of the group of all rotations, $\mathrm{SO}_{3},[21,27,42]$ can be extended to the IRs of lower point groups, which is important in the potential extension of the spherical wave description of EMR to molecular problems. We expand upon this point in Section 5 of this paper.

\subsection{A note on the issue of gauge invariance}

All theories of radiation-matter interactions should be gauge invariant. While this is not key to this review, readers concerned with the deeper underlying principles of the QED description of the interaction of electromagnetic radiation with atoms and molecules may be concerned to know whether the expressions which we describe and apply here are gauge 
invariant. Griffith [77, p. 43] has examined this problem in detail and has shown, in very general terms, that the wavefunctions are changed only by a phase factor when the interaction Hamiltonian, has been modified by the addition of the gauge function to the scalar and vector potentials. Such a transformation therefore makes no difference at all to the observable results of the calculation. Moreover, Griffith has also proved [appendix 5 of reference 77] for the Coulomb gauge, which we also use here, that the first and second order perturbation expressions which arise in applications of the theory are also gauge invariant. In that context he makes the important observation that when applying the second-order perturbation theory it is essential to consider the second-order term which arises in the firstorder calculation. The question of gauge invariance within the context of multipole transitions is addressed by Grant, ${ }^{47}$ while Glushkov et al. addresses relativistic corrections within a gauge invariant context. ${ }^{48}$

\section{The different descriptions of light for atomic interactions}

\subsection{Term-by-term comparison of PW and SW descriptions of light}

Our discussion begins with an examination of the individual terms in the expansions (19) and (20) as they are applied, in particular, to the calculation of the transition moment between atomic or molecular electronic states [49]. Oúr perspective is from the point-ofview of molecular quantum electrodynamics, $[16,50]$ whereby we focus on the calculation of quantum amplitudes between initial and final states, and hence it is sufficient to consider time-independent standing waves in the z-direction for which Eq. (19) becomes:

$$
\hat{\vec{e}}_{1 n} \exp [\mathrm{i} k z]=\hat{\vec{e}}_{1 n}\left[1+\mathrm{i} k z-\frac{(k z)^{2}}{2 !}-\frac{\mathrm{i}(k z)^{3}}{3 !}+\cdots\right]
$$

and expression (20) reduces [20] to:

$$
\hat{\vec{e}}_{1 n} \exp [\mathrm{i} k z]=\hat{\vec{e}}_{1 n} \sum_{l} \mathrm{i}^{l}(2 l+1) j_{l}(k r) P_{l}(\cos \vartheta)
$$

In expression (20a), $P_{l}(\cos \vartheta)$ is Legendre polynomial with $\vartheta=\cos ^{-1}(z / r)$ and $j l(k r)$ is the spherical Bessel function of half-integral order appropriate for the description of standing waves [49]. This is the form of Bessel function required to calculate the transition moment between two electronic states and hence the transition probability and rate. 
In the context of transition moments, expressions (19) and (19a) are particularly important in that successive terms are used as the basis for assigning the order of polarity of the radiation involved in the transition. In particular, when $k z$ is rapidly diminishing (i.e. $k z$ $<<1$ ), the different terms are used to define the type of transition, i.e. dipolar, quadrupolar, octapolar, etc $[52,32]$. Thus, if the first term in the series provides a coupling between two atomic or molecular electronic states, with a consequent absorption or emission of radiation, that radiation is said to be electric dipolar (E1) radiation, and the transition between the two states is an electric dipole transition. If it is necessary to go to the second term before a nonzero coupling is found, then the associated radiation is electric quadrupolar (E2) and/or magnetic dipolar (M1) radiation and the transition is described as an electric quadrupole or magnetic dipole transition; or as an indeterminate combination of the two. Higher moments appear to have been discussed only very rarely in atomic and molecular physics, e.g. [53], though more commonly in nuclear gamma-ray spectroscopy [54]. Since the multipolarity is intimately connected with the angular momentum (AM) of the radiation and the coupled states, the assignment of polarity (i.e. the order of truncation of the multipole) also implies an assignment of AM to the transition and the radiation.

In the case of expressions (20) and (20a), the AM of the radiation is given immediately by the parameters of the VSH in the form [42]:

$$
\hat{J}\left|J l M>=J(J+1) \hbar^{2}\right| J l M>
$$

and

$$
\hat{J}_{Z}|J l M>=M \hbar| J l M>
$$

Since both expressions, (19) and (20), assign polarity and AM to their successive terms, it is useful to determine under what conditions there is term-by-term equality of the two series. For that purpose it is sufficient to compare the right-hand sides of (19a) and (20a), neglecting the polarization yector. Recalling that $z=r \cos \vartheta$, we find the following expressions for the first few terms in the expansion of (20a):

$$
\begin{gathered}
l=0 \rightarrow 1-\frac{(k r)^{2}}{3 !}+\frac{(k r)^{4}}{5 !}-\frac{(k r)^{6}}{7 !}+\cdots \\
l=1 \rightarrow \mathrm{i} k z-\frac{3 \mathrm{i} k z(k r)^{2}}{5 \times 3 !}+\frac{3 \mathrm{i} k z(k r)^{4}}{7 \times 5 !}-\cdots \\
\sim 11 \sim
\end{gathered}
$$




$$
l=2 \rightarrow-\frac{(k z)^{2}}{2 !}+\frac{(k r)^{2}}{3 !}+\frac{30(k z)^{2}(k r)^{2}}{7 \times 5 !}-\cdots
$$

Comparison of equations (23a-c) with (19a) now shows that the both the PW and SW series tend to term-by-term equality, when the second and subsequent terms in the PW series are neglected, i.e. as $k r \rightarrow 0$ (the long-wavelength approximation). This is precisely the criterion, in electronic spectroscopy, upon which the assignment of polarity and AM to the individual terms of equation 1 rests $[55,32]$.

Motivated by the specific results above, we now assume that $k r<<1$ which allows us to describe a more general analysis by approximating the spherical Bessel function as:

$$
j_{l}(k r) \cong \frac{(k r)^{l}}{(2 l+1)(2 l-1)(2 l-3) \cdots 1} \equiv \frac{(k r)^{l}}{(2 l+1) ! !}
$$

Expressing $j_{l}(k r)$ in this way and expanding the Legendre polynomial we obtain from equation (20a) the expression:

$$
\begin{aligned}
\exp (\mathrm{i} k r)= & \sum_{l} \frac{1}{2^{l}(2 l-1) ! !} \sum_{m=0}^{\leq\left(\frac{l}{2}\right)}(-1)^{m} \frac{(2 l-2 m) !}{(l-m) !(l-2 m) ! m !}(\mathrm{i} k z)^{l}\left(\frac{r}{z}\right)^{2 m} \\
& \left.\equiv \sum_{l} \sum_{m=0}^{\leq\left(\frac{l}{2}\right)} \Omega_{l, m}\right)
\end{aligned}
$$

Table 1 lists some early terms in this expression, and we note that the terms in the $m=0$ column are precisely those of expansion (19a), but that higher terms are present for $l \geq 2$.

Table 1. Terms $\Omega_{l, m}$ in Equation 25

\begin{tabular}{|l|c|c|c|}
\hline & $m=0$ & $m=1$ & $m=2$ \\
\hline$l=0$ & 1 & & \\
\hline$l=1$ & $+\mathrm{i} k z$ & & \\
\hline$l=2$ & $-(1 / 2)(k z)^{2}$ & $+(1 / 6)(k r)^{2}$ & \\
\hline
\end{tabular}




\begin{tabular}{|l|c|c|c|}
\hline$l=3$ & $-(\mathrm{i} / 6)(k z)^{3}$ & $+(\mathrm{i} / 10)(k z)(k r)^{2}$ & \\
\hline$l=4$ & $+(1 / 24)(k z)^{4}$ & $-(1 / 28)(k z)^{2}(k r)^{2}$ & $+(1 / 280)(k r)^{4}$ \\
\hline$l=5$ & $+(\mathrm{i} / 120)(k z)^{5}$ & $-(\mathrm{i} / 108)(k z)^{3}(k r)^{2}$ & $+(\mathrm{i} / 504)(k z)(k r)^{4}$ \\
\hline
\end{tabular}

When considering the emission of a photon as a consequence of a change of state of an atom, it appears intuitively reasonable to regard Eq. (20), based as it is upon VSH's centred at the atomic nucleus, as the most suitable description of the very early stages of that process. Naturally, by the time the emitted photon has travelled a few nanometres from the atom and, a fortiori, has reached any physically realisable measuring instrument, the corresponding electromagnetic wave can be regarded, to a very good approximation, as a plane wave as described by Eq. (19). However, as $r$, the distance of the emitted photon from the emitter, approaches $\lambda$, the wavelength of the emitted light, the assumption that $k r \approx 0$ becomes less valid, the terms for $m>0$ can no longer be neglected and the term-by-term equality of the Eq. (19) and (20) is lost. But the exact value of the photon AM, given by the quantum number $J$ of the VSH, $|J, l, n(\vartheta, \varphi)\rangle$, of Eq. (20), is unaffected by increasing $r$. This implies that the individual terms of expression (19) can no longer be regarded as precise descriptions of the photon AM and that this description of the AM carried by the photon is progressively degraded as the distance of the photon from its source increases. This result has a clear resonance with the work of Andrews [56] who has concluded, as a result of a PW-based analysis, that '... any photon emitted by multipolar decay, with the sole exception of dipole emission, carries away from its source an angular momentum that acquires as it propagates beyond the near-zone a progressively broader spectrum of integer values'. This suggests that the angular momentum of the photon becomes increasingly undefined as it moves away from its source. But the implication of the SW results is not that the angular momentum of the photon changes; but rather that the description provided by the PW analysis becomes less valid as $r$ increases.

The exception of dipole emission noted by Andrews would appear, in the present interpretation, to be a consequence of the fact that, within the limits set by the assumption $k r \ll<$, the dipole term (23a) has no dependence on $r$ (or $z$ ), whereas the subsequent terms (23b and 23c) do.

\subsection{Selection rules and transition moments}


Clearly, the most important test in comparing the PW and SW descriptions of EMR is to demonstrate that the methods give the same quantitative results. This has been done in detail in the case of atomic transition moments [49]. Though the results of the SW and PW calculation of atomic transition moments are in exact agreement, they do raise a very fundamental question: How do the results differ with regard to a particular multipole transition? That is to say, can one expect to get convergence of results between the two mathematical descriptions of the photonic fields for dipolar, quadrupolar, octupolar, ... emission. In fact it has been shown that there is a lack of one-to-one correspondence of the consecutive terms in the expansions (19) and (20), [49] presumably indicating that at least one of the descriptions is inadequate for describing the emission from a particular $l$-pole.

As a specific illustration, consider the calculation of the transition moment matrix element, $T$, for the stimulated electronic transition from the 1s atomic orbital of hydrogen to the $2 \mathrm{p}_{+1}$. Both calculations lead to exactly the same algebraic expression $(26)$ for $T[49,16]$ :

$$
\begin{gathered}
T \equiv\left\langle 2 p_{+1} ;(n-1)\left(k, e_{1+1}\right)\left|\left(\frac{e}{m}\right) \vec{A} \cdot \vec{p}\right| 1 s, n\left(k, e_{1+1}\right)\right\rangle \\
=-N_{1 s} N_{2 p}\left(\frac{n \hbar}{2 \varepsilon_{o} c k V}\right)^{1 / 2} \cdot \frac{\mathrm{i} \hbar \pi}{m} \cdot \frac{16 \sqrt{2}}{3}\left(\frac{2 a_{0}}{3}\right)^{3} \sum_{0}^{\infty}(-1)^{n}(n+1)\left(\frac{2 k a_{0}}{3}\right)^{2 n} .
\end{gathered}
$$

Here, $N_{1 \mathrm{~s}}$ and $N_{2 \mathrm{p}}$ are the normalization factors for the hydrogen $1 \mathrm{~s}$ and $2 \mathrm{p}$ orbitals respectively, $\vec{A}$ the vector potential associated with the photon, $\vec{p}$ the linear momentum operator of the electron, $k$ the wavenumber, $V$ the quantization volume, $a_{0}$ the Bohr radius, $c$ the velocity of light, $m$ the mass and $e$ the charge of the electron.

It is important to note that the exact equality of the PW and SW results is only found when the complete PW expansion of the exponential is used, not merely the first term as is normally the case when using the long-wavelength approximation. The difference between the full and approximate PW result is described clearly by Craig and Thirunamachandran [16, page 105].

However, though the algebraic results are identical, as of course they must be, the interpretation of the terms in the summation is not and can lead to ambiguity when considering the multipolar contributions to the emission process. In the case of the PW calculation, the terms in the sum arise from the successive terms in the expansion of the exponential in Eq. (19), and can therefore, for consistency with the definition of dipolar, 
quadrupolar,... transitions, be regarded as representing small multipolar corrections to the dominant $(n=0)$ dipolar term. But, in the SW calculation the identical series arises as the summed results of integrating two expressions of the form, [49]

$\int_{0}^{\infty} \exp \left(-\frac{r}{\alpha}\right) j_{n}(k r) r^{3} d r$

with $n=0$ and 2 and $\alpha=2 a_{0} / 3$, each of which corresponds to an uptake of exactly $\sqrt{2} \hbar$ units of angular momentum by the atom, i.e. an E1 transition. Accordingly, there is not a one-toone correspondence between the definitions of multipolar transitions in the PW and SW descriptions though, as we have shown above, the correspondence becomes increasingly close as $k r \rightarrow 0$. This is quite consistent with the general ethos of the application of the PW theory to the calculation of transition probabilities in atomic spectroscopy where the longwavelength approximation is almost invariably applied. The neglect of/higher terms in the expansion 1 also accounts for the combining of E2 and M1 transitions in the PW description, whereas they are rigorously separated in the SW calculation where the long-wavelength approximation offers no computational advantage.

\subsection{The polarisation of atomic emission spectra}

A PW solution of this problem was published by Rubinowicz in 1929/30 [57]. Condon and Shortley [55] describe the application to quadrupole transitions in some detail and Huff and Houston give the results for octupoles [58].

In the SW method [44] the parameters $(J, l, M)$ of the VSH description of the photon emitted in any particular atomic process are determined by the selection rules for that transition [59]. The VSH, $\mid J, l, M>$, is then expressed in terms of the unit vectors of the spherical polar co-ordinate system, $\widehat{\vec{R}}, \widehat{\vec{\Theta}}$, and $\widehat{\vec{\Phi}}$ in the form:

$$
\mid J, l, M>=\Theta_{J, l, M} \widehat{\widehat{\Theta}}+\Phi_{J, l, M} \widehat{\widehat{\Phi}}+R_{J, l, M} \widehat{\vec{R}}
$$

where $\Theta_{J, l, M}, \Phi_{J, l, M}$ and $R_{J, l, M}$ are complex numerical coefficients which are simple functions of $J, l$ and $M$. When this has been done, if the imaginary part of the quotient $\Phi_{J, l, M} / \Theta_{J, l, M}$ is negative we have emission of left elliptically polarized light (LEP) and when it is positive, right elliptically polarized light (REP) [49]. When $\operatorname{Im}\left\{\Phi_{J, l, M} / \Theta_{J, l, M}\right\}= \pm 1$ the emitted light is circularly polarized. At the extremes; if the quotient is infinite we have light linearly 
polarized along $\widehat{\widehat{\Phi}}$, designated $\sigma$ in atomic spectroscopy, and when it is zero we have linear polarization along $\widehat{\widehat{\Theta}}$, i.e. $\pi$. General expressions for the coefficients $\Theta_{J, l, M}, \Phi_{J, l, M}$ and $R_{J, l, M}$ are available as simple functions of the three parameters, $J, l$ and $M$, and the direction in which the photon is emitted (values of $\vartheta$ and $\varphi$ ) [44] and it is therefore an easy task to investigate the emission for any transition, even where there are large changes of AM. The problem is much more difficult in the case of a PW analysis following Rubinowicz [57] and, to our knowledge, no general expressions for that purpose have been published.

\subsection{The calculation of $\mathbf{E}$ - and B-fields of a multipolar source}

A PW examination of electric and magnetic fields from dipolar and quadrupolar sources has been described by Rice et al., [60] who calculated their interaction with a test dipole. They were able to obtain results of a very general nature, it being only necessary to specify the nature of the source, i.e. dipole or quadrupole. The SW approach requires a more detailed model so, in order to make a comparison, we specify a quadrupolar source comprising four charges, oscillating between $+q$ and $-q$, and positioned as follows at $x$ and $z$ co-ordinates $(q, x, z):(+q,+1 / 2 \mathrm{~s},+1 / 2 \mathrm{~s}) ;(-q,-1 / 2 \mathrm{~s},+1 / 2 \mathrm{~s}) ;(+q,-1 / 2 \mathrm{~s},-1 / 2 \mathrm{~s}) ;(-q,+1 / 2 \mathrm{~s},-1 / 2 \mathrm{~s})$. Such a model might represent the transition electron density of an atomic $1 \mathrm{~s} \leftarrow 3 \mathrm{~d}_{\mathrm{xz}}$ transition.

In the SW description the longitudinal or radial $E$-field is directed along the unit vector $\hat{\vec{R}}$ [56],

$$
\begin{gathered}
E_{\|}=\frac{3 \mathrm{i} q s^{2} k^{4}}{40 \pi \varepsilon_{o}}\left\{h_{1}^{(1)}(k R)+h_{3}^{(1)}(k R)\right\} \sin 2 \vartheta \cos \varphi \hat{\vec{R}} . \\
=\frac{3 \mathrm{i} q s^{2} k^{4}}{8 \pi \varepsilon_{o}} \exp (\mathrm{i} k r)\left\{\frac{\mathrm{i}}{(k R)^{2}}-\frac{3}{(k R)^{3}}-\frac{3 \mathrm{i}}{(k R)^{4}}\right\} \sin 2 \vartheta \cos \varphi \hat{\vec{R}} .
\end{gathered}
$$

In the PW description, for the $i^{\text {th }}$ Cartesian component of the parallel E-field of a general quadrupolar emission we have, [60],

$$
E_{\|}=\frac{3 \mathrm{i} k^{4}}{4 \pi \varepsilon_{o}} \exp (\mathrm{i} k r) Q_{j k} \hat{R}_{i} \hat{R}_{j} \hat{R}_{k}\left\{\frac{\mathrm{i}}{(k R)^{2}}-\frac{3}{(k R)^{3}}-\frac{3 \mathrm{i}}{(k R)^{4}}\right\}
$$

where the tensor convention of summation over the repeated suffixes $j$ and $k$ is implied. 
In order to make a comparison conversion from Cartesian to polar co-ordinates is first required, and for an out-going wave we have:

$\widehat{\vec{\Theta}}=\cos \vartheta \cos \varphi \hat{\vec{x}}+\cos \vartheta \sin \varphi \hat{\vec{y}}-\sin \vartheta \hat{\vec{z}}$

$\widehat{\vec{\Phi}}=-\sin \vartheta \hat{\vec{x}}+\cos \varphi \hat{\vec{y}}$

$\widehat{\vec{R}}=\sin \vartheta \cos \varphi \hat{\vec{x}}+\sin \vartheta \sin \varphi \hat{\vec{y}}+\cos \vartheta \hat{\vec{z}}$.

Various different formulas for calculating the quadrupole moment of a charge distribution can be found in the literature. Rice et al. [60] employed Eq. (32) which is the form appropriate for that calculation [71].

$$
Q_{\alpha \beta}=\frac{1}{6} \sum_{n}\left(3 \alpha_{n} \beta_{n}-r_{n}^{2} \delta_{\alpha \beta}\right) q_{n}
$$

Using Eq. (32) we find that the only non-vanishing quadrupole moments are $Q_{\mathrm{xz}}=Q_{\mathrm{zx}}=$ $1 / 2 q s^{2}$, giving:

$Q_{j k} \widehat{\vec{R}}_{i} \widehat{\vec{R}}_{j} \widehat{\vec{R}}_{k}=Q_{x z}\left\{\frac{2 x^{2} z}{r^{3}} \hat{\vec{x}}+\frac{2 x y z}{r^{3}} \hat{\vec{y}}+\frac{2 x z^{2}}{r^{3}} \hat{\vec{z}}\right\}=1 / 2 q s^{2} \sin 2 \vartheta \cos \varphi \hat{\vec{R}}$.

And,

$E_{||}=\frac{3 \mathrm{i} q s^{2} k^{4}}{8 \pi \varepsilon_{o}} \exp (\mathrm{i} k r)\left\{\frac{\mathrm{i}}{(k R)^{2}}-\frac{3}{(k R)^{3}}-\frac{3 \mathrm{i}}{(k R)^{4}}\right\} \sin 2 \vartheta \cos \varphi \hat{\vec{R}}$

incomplete agreement with the SW result.

For the transverse E-field in the SW formulation we have [49],

$$
\begin{gathered}
E_{\perp}=\frac{\mathrm{i} q s^{2} k^{4}}{40 \pi \varepsilon_{o}}\left\{3 h_{1}^{(1)}(k R)-2 h_{3}^{(1)}(k R)\right\}\{\cos 2 \vartheta \cos \varphi \widehat{\vec{\Theta}}-\cos \vartheta \sin \varphi \widehat{\vec{\Phi}}\} \\
=\frac{\mathrm{i} q s^{2} k^{4}}{8 \pi \varepsilon_{o}} \exp (\mathrm{i} k r)\left\{-\frac{1}{k R}-\frac{3 \mathrm{i}}{(k R)^{2}}+\frac{6}{(k R)^{3}}+\frac{6 \mathrm{i}}{(k R)^{4}}\right\}\{\cos 2 \vartheta \cos \varphi \widehat{\vec{\Theta}}-\cos \vartheta \sin \varphi \widehat{\vec{\Phi}}\}
\end{gathered}
$$

And for the $i^{\text {th }}$ component of that field in the PW formulation [58] 


$$
E_{\perp i}=-\frac{\mathrm{i} k^{4}}{8 \pi \varepsilon_{o}} \exp (\mathrm{i} k r) Q_{j k} \hat{\vec{R}}_{k}\left(\hat{\vec{R}}_{i} \hat{\vec{R}}_{j}-\delta_{i j}\right)\left\{-\frac{1}{k R}-\frac{3 \mathrm{i}}{(k R)^{2}}+\frac{6}{(k R)^{3}}+\frac{6 \mathrm{i}}{(k R)^{4}}\right\}
$$

where summation over repeated suffixes is again implied.

Conversion from Cartesian to polar co-ordinates gives,

$$
\begin{gathered}
Q_{j k} \widehat{\vec{R}}_{k}\left(\hat{\vec{R}}_{i} \hat{\vec{R}}_{j}-\delta_{i j}\right)=\left\{\left[\frac{2 x^{2} z}{r^{3}}-\frac{z}{r}\right] \widehat{\vec{x}}+\left[\frac{2 x y z}{r^{3}}\right] \widehat{\vec{y}}+\left[\frac{2 x z^{2}}{r^{3}}-\frac{x}{r}\right] \hat{\vec{z}}\right\}^{\prime} \\
=Q_{x z}\{-\cos 2 \vartheta \cos \varphi \widehat{\widehat{\Theta}}+\cos \vartheta \sin \varphi \widehat{\vec{\Phi}}\}
\end{gathered}
$$

and, since the only non-vanishing quadrupole moments are $Q_{\mathrm{xz}}=Q_{\mathrm{zx}}$, we now have:

$$
\begin{gathered}
E_{\perp}=-\frac{\mathrm{i} k^{4}}{4 \pi \varepsilon_{o}} \exp (\mathrm{i} k r) Q_{x z}\left\{-\frac{1}{k R}-\frac{3 \mathrm{i}}{(k R)^{2}}+\frac{6}{(k R)^{3}}+\frac{6 \mathrm{i}}{(k R)^{4}}\right\} \times \\
\{\cos 2 \vartheta \cos \varphi \widehat{\vec{\Theta}}-\cos \vartheta \sin \varphi \widehat{\vec{\Phi}}\},
\end{gathered}
$$

and inserting the value of $Q_{x z}$ from above:

$$
\begin{gathered}
E_{\perp}=-\frac{\mathrm{i} q s^{2} k^{4}}{8 \pi \varepsilon_{o}} \exp (\mathrm{i} k r)\left\{-\frac{1}{k R}-\frac{3 \mathrm{i}}{(k R)^{2}}+\frac{6}{(k R)^{3}}+\frac{6 \mathrm{i}}{(k R)^{4}}\right\} \times \\
\{\cos 2 \vartheta \cos \varphi \widehat{\vec{\Theta}}-\cos \vartheta \sin \varphi \widehat{\vec{\Phi}}\} .
\end{gathered}
$$

As for $\boldsymbol{E}_{\|}$, in complete quantitative agreement with the SW result.

\section{Molecular interactions and resonance energy transfer}

\subsection{Electronic couplings}

In the previous section, the focus was on atomic radiative processes. Now we consider general molecular/systems that can be orientated in space, with respect to each other and a coordinate system. The ability to express easily the fully-retarded electric or magnetic field of a specific multipole, as illustrated in Section 3, also offers a route to the calculation of multipole-multipole interactions. In particular resonance energy transfer (RET), which involves the movement of an electronically excited state from a donor molecule to an acceptor molecule. There have been many studies of these problems within 
a quantum electrodynamical context; notably by Avery [62], Power and Thirunamachandran [63-65], Craig and Thirunamachandran [16] and later by Andrews, Salam, and others [50, 66-71].

Quantum amplitudes, which characterize interactions between electronic species with a quantum electrodynamical context, are strongly dependent on the distance between chromophores, as well as the relative orientation of their transition moments. In the case of RET, the matrix element defining the electronic coupling between molecules is found to be $[16,50]$,

$$
V_{i j}^{ \pm}(k, \vec{R})=\sigma_{i j}(k, \vec{R})+i \tau_{i j}(k, \vec{R})
$$

where

$$
\begin{aligned}
& \sigma_{i j}(k, \vec{R})=\left(4 \pi \varepsilon_{0} R^{3}\right)^{-1}\left\{\left(\cos k R+k R \operatorname{sink} R\left[\delta_{i j}-3 \hat{R}_{i} \hat{R}_{j}\right]-k^{2} R^{2} \cos k R\left[\delta_{i j}-\hat{R}_{i} \hat{R}_{j}\right]\right)\right\} \\
& \tau_{i j}^{ \pm}(k, \vec{R})=\left(4 \pi \varepsilon_{0} R^{3}\right)^{-1}\left\{\mp\left(\sin k R-k R \cos k R\left[\delta_{i j}-3 \hat{R}_{i} \hat{R}_{j}\right] \pm k^{2} R^{2} \sin k R\left[\delta_{i j}-\hat{R}_{i} \hat{R}_{j}\right]\right)\right\}
\end{aligned}
$$

Different approaches have been adopted in deriving this equation, including contour integration within the complex plane [66], and the use of judicious substitutions [71]. In both cases, non-physical, as well as the correct solutions emerge from the calculation. This lead to ambiguity in the sign of the imaginary part of the quantum amplitude Eq. (40). Andrews et al. point out that as it is the square of a matrix element that is associated with a quantum mechanical observable, ascribing the sign to a particular process (emission or absorption) is a moot point. Interestingly, because the integral is completely absent from the SW calculation the ambiguity in the sign of the imaginary contribution to the coupling is immediately seen to be simply related to the choice of in-coming or out-going waves and the time-dependence factor of the radiation; $\exp (+\mathrm{i} \omega t)$ or $\exp (-\mathrm{i} \omega t)$.

The final results of the SW calculation are in complete agreement with the particular PW result, which is now accepted as correct [71]. Furthermore, the SW theory, being expressed in spherical polar co-ordinates, intrinsically separates the transverse components of the photon's fields directed along $\Theta$ and $\Phi$ from the radial or longitudinal factors directed along $R$ and thus clarifies the orientational dependence of the interaction. Therefore, since only the latter, the radial terms, are affected by retardation, the transverse terms can be clearly related to the classical electrodynamic expressions, in which retardation does not 
feature, and use can be made of this connection in many calculations as we shall illustrate below.

Comparison of expansions (19) and (20) immediately reveals some of the relative strengths and weaknesses of the PW and SW descriptions of electromagnetic radiation and its interaction with matter. One of the most important of these is the clear separation of longitudinal (radial) and transverse components, which is intrinsic to the $\mathrm{SW}$ description because of the use of polar co-ordinates. That is to say, if we envisage a series of detectors isotropically arranged around a source, and a photon is detected at a fixed distance, $\vec{R}$, from the source, we can use the coordinate system defined by the photon. This makes the SW coordinate system more versatile for photon counting experiments, where directionality of the photon is important. An example of where this may be important is in mapping emission profiles for a particular transition, for example in identifying near field effects of nanoantennas, where higher order multi-poles may play a role. [72]

\subsection{Dipole-dipole interaction}

Because, in the spherical wave analysis, the interaction separates naturally into transverse $\left(M_{R}^{\prime}, M \Theta^{\prime}\right.$ and $\left.M \Phi^{\prime}\right)$ and longitudinal (functions of $k R$ ) parts we can, write the real and imaginary parts of a dipole-dipole interaction, $W$, in the form [73]:

$$
\begin{aligned}
W(R e) & =-\frac{k^{3}}{4 \pi \varepsilon_{o}}\left\{2 \mathrm{M}_{R}^{\prime}+\mathrm{M}_{\Theta}^{\prime}+\mathrm{M}_{\Phi}^{\prime}\right\}\left[\frac{\sin k R}{(k R)^{2}}+\frac{\cos k R}{(k R)^{3}}\right]+\frac{k^{3}}{4 \pi \varepsilon_{o}}\left\{\mathrm{M}_{\Theta}^{\prime}+\mathrm{M}_{\Phi}^{\prime}\right\}\left[\frac{\cos k R}{k R}\right] \\
= & -\frac{k^{3}}{4 \pi \varepsilon_{o}}\left\{\left[2 \mathrm{M}_{R}^{\prime}+\mathrm{M}_{\Theta}^{\prime}+\mathrm{M}_{\Phi}^{\prime}\right]\left[\frac{\sin k R}{(k R)^{2}}+\frac{\cos k R}{(k R)^{3}}\right]-\left[\mathrm{M}_{\Theta}^{\prime}+\mathrm{M}_{\Phi}^{\prime}\right]\left[\frac{\cos k R}{k R}\right]\right\},
\end{aligned}
$$

and

$$
\begin{aligned}
W(I m) & =-\frac{k^{3}}{4 \pi \varepsilon_{o}}\left\{2 \mathrm{M}_{R}^{\prime}+\mathrm{M}_{\Theta}^{\prime}+\mathrm{M}_{\Phi}^{\prime}\right\}\left[-\frac{\cos k R}{(k R)^{2}}+\frac{\sin k R}{(k R)^{3}}\right]+\frac{\mathrm{i} k^{3}}{4 \pi \varepsilon_{o}}\left\{\mathrm{M}_{\Theta}^{\prime}+\mathrm{M}_{\Phi}^{\prime}\right\}\left[\frac{\sin k R}{k R}\right] \\
& =-\frac{k^{3}}{4 \pi \varepsilon_{o}}\left\{\left[2 \mathrm{M}_{R}^{\prime}+\mathrm{M}_{\Theta}^{\prime}+\mathrm{M}_{\Phi}^{\prime}\right]\left[-\frac{\cos k R}{(k R)^{2}}+\frac{\sin k R}{(k R)^{3}}\right]-\left[\mathrm{M}_{\Theta}^{\prime}+\mathrm{M}_{\Phi}^{\prime}\right]\left[\frac{\sin k R}{k R}\right]\right\} .
\end{aligned}
$$


From this we see that for $k R \approx 0$ (very long wavelength or very small $R$ ) $\cos k R \approx 1$ and $\sin k R \approx k R$. And then

$$
-\frac{\cos k R}{(k R)^{2}}+\frac{\sin k R}{(k R)^{3}} \approx-\frac{1}{(k R)^{2}}+\frac{1}{(k R)^{2}} \approx 0
$$

and the imaginary contribution to the interaction is approximately:

$$
W(I m) \approx \frac{k^{3}}{4 \pi \varepsilon_{o}}\left[\mathrm{M}_{\Theta}^{\prime}+\mathrm{M}_{\Phi}^{\prime}\right]\left[\frac{\sin k R}{k R}\right] \approx \frac{k^{3}}{4 \pi \varepsilon_{o}}\left[\mathrm{M}_{\Theta}^{\prime}+\mathrm{M}_{\Phi}^{\prime}\right]
$$

i.e. a transverse interaction with a very small dependence upon $R$ !

This formulation is also valuable in that it allows a ready exploration of the relative significance of the two contributions. The longitudinal component tends to be neglected in far-field applications, though it can be very important where the coupling chromophores are close together. The case of anthracene provides an interesting example. In solution, the molecule shows a strong $\pi^{*} \leftarrow \pi$ transition at ca. $250 \mathrm{~nm}$ with an oscillator strength which corresponds to a transition dipole moment of $3.7 \times 10^{-29} \mathrm{Cm}$. In the crystal there are two molecules per unit cell and the interaction between them splits this transition into two bands; the Davydov splitting. This was studied by Craig and Hobbins [74] who used their experimental data on the splitting, and especially the polarization of the resulting bands, to assign the transition to the long-axis polarized $B_{3 u} \leftarrow A_{g}$ (in $D_{2 h}$ ) transition of the molecule. Unfortunately, the peak of the higher-energy band of the Davydov pair could not be identified since it lay outside of the range of possible measurement. Furthermore, since the absorption bands are very broad and complicated by numerous other effects no precise measurement of the magnitude of the splitting is possible, even if measurements in the required region are made. But in a further paper on this subject [75], Craig notes that the Davydov splitting of the intense transitions of the aromatic hydrocarbons, “... may amount to some thousands of wavenumbers ...". In our calculations we find an interaction of $\sim 15 \times 10^{-3} \mathrm{E}_{\mathrm{h}} \approx 3,300 \mathrm{~cm}^{-1}$ which would produce a splitting of ca. $6,600 \mathrm{~cm}^{-}$ 1. This may be an overestimate, but the important point in the present context is the following. 
The centres of the two molecules in the unit cell are $51.8 \mathrm{~nm}$ apart and the two transition dipoles lie at $\sim 61^{\circ}$ and $\sim 70^{\circ}$ to the line of centres and subtend an angle of $\sim 15^{\circ}$. When separated into parallel and transverse parts it is found that the longitudinal field contributes almost as much to the splitting as the transverse; $44 \%$ and $56 \%$ respectively. And calculations for increased intermolecular distances show that the transverse field coupling, though dominant at large $r$, does not rise above twice the longitudinal-field coupling until $r / \lambda$ exceeds 0.3 .

This persistence of the effect of the longitudinal field can be even more marked. In some cases which we have studied the coupling due to the transverse field does not significantly exceed that due to the longitudinal field until $r / \lambda$ exceeds 0.8 .

\subsection{Dipole-quadrupole interaction}

Since only the radial terms are affected by retardation, the transverse terms can be easily related to the classical electrodynamic expressions and in many cases use can be made of this connection. Indeed, it is immediately clear from classical electrodynamics that, in the case of the calculation of dipole-quadrupole interaction two theoretical approaches are available. One is based upon the energy, $W$, of a dipole, $\mu$, in the field of a quadrupole, $E^{Q}$, and the other upon the equation given by Jackson [61] for the energy, $W$, of a quadrupole, $Q_{\alpha \beta}$, in the gradient of the field of a dipole, $E^{D}$, Eq. (45).

$$
W=-\frac{1}{6} \sum_{\alpha} \sum_{\beta} Q_{\alpha, \beta} \frac{\partial E_{\alpha}^{D}}{\partial \beta}
$$

This flexibility could prove useful in practical problems. In order to illustrate the wider possibilities of the SW description we now outline the calculation of the interaction between a dipole and a quadrupole by these two methods.

For the calculation of the energy of a dipole in the electromagnetic field of a quadrupole we first need to define the quadrupoles. Six quadrupoles are specified in order to accommodate all six components of the quadrupole moment tensor, which is assumed to be symmetric, i.e. $Q_{\alpha \beta}=Q_{\beta \alpha}$. They are of two types. Square planar, $\mathrm{XY}, \mathrm{XZ}$ and YZ with, in each case, a charge of $+q$ in the positive quadrant of the axes specified. XY is illustrated below. Linear, $\mathrm{X}^{2}, \mathrm{Y}^{2}$ and $\mathrm{Z}^{2}$ with charges disposed as for the $\mathrm{Z}^{2}$ illustrated in Figure 1. The centre of each quadrupole lies at the origin of co-ordinates. 

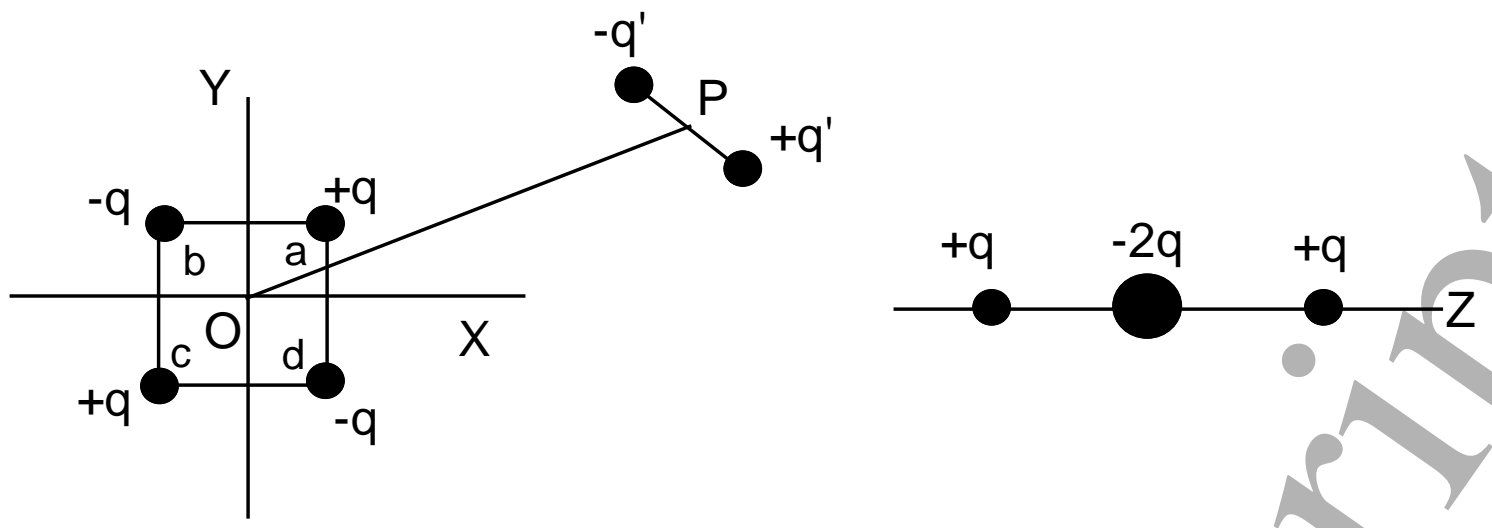

Figure 1. Illustrative Dipoles and Quadrupoles.

Here, the quadrupole moments are calculated using the formula used by Scholes and Andrews, [69]:

$$
Q_{\alpha \beta}=\frac{1}{2} \sum_{n} \alpha_{n} \beta_{n} q_{n}
$$

This equation was used for a PW calculation, the results of which we to compare our SW results, below. The non-zero quadrupole moments found using Eq. (46) can be found in Table A1, where $s$ is the distance of each individual charge centre from the quadrupole centre at the origin. The centre of the dipole lies at the point $\mathrm{P}$ designated by the polar coordinates $r, \vartheta$ and $\varphi$, and the distance between the two charges, $\pm q^{\prime}$, is $l$ so that the dipole moment $\mu=\mathrm{q}^{\prime} l$. The dipole is specified in terms of its magnitude and orientation, or of its Cartesian components.

In the case of the dipole in the field of the quadrupole, the interaction energy, $W$, is obtained by taking the negative of the scalar product of the vectors $\vec{\mu}$ and $\vec{E}$ :

$$
W=-\vec{\mu} \cdot \vec{E}=-\left[\mu_{R} E_{R}+\mu_{\Theta} E_{\Theta}+\mu_{\Phi} E_{\Phi}\right]
$$

The quadrupole fields are obtained in terms of the polar vectors, $\vec{\Theta}, \vec{\Phi}$ and $\vec{R}$, and hence we express the Cartesian dipole components in the same manner. For a wave out-going from the quadrupole $\mu \Theta, \mu \Phi$ and $\mu_{R}$ are related to $\mu_{\mathrm{x}}, \mu_{\mathrm{y}}$ and $\mu_{\mathrm{z}}$ by the usual transformation between the two coordinate systems:

$$
\mu_{\Theta}=\cos \vartheta \cos \varphi \mu_{x}+\cos \vartheta \sin \varphi \mu_{y}-\sin \vartheta \mu_{z}
$$




$$
\begin{gathered}
\mu_{\Phi}=-\sin \varphi \mu_{x}+\cos \varphi \mu_{y} \\
\mu_{R}=\sin \vartheta \cos \varphi \mu_{x}+\sin \vartheta \sin \varphi \mu_{y}+\cos \vartheta \mu_{z}
\end{gathered}
$$

The quadrupole fields, $\vec{E}_{Q}$, may be determined in the way described previously. The components can be found in Table A2.

This result clearly separates the radial (longitudinal, $F_{\|}$) term from the tran'sverse $\left(F_{\perp}\right)$ term, and the greater "reach" of the latter is already apparent in that it contains the only $(k r)^{-1}$ contribution to the coupling. This clear separation, which is less clear in the $P W$ description, is one of the most useful aspects of the spherical wave approach.

A separation of these expressions into real and imaginary parts is also of interest; we find:

$$
\begin{aligned}
& \operatorname{Re}\left[F_{\|}(r)\right]=-\frac{\sin k r}{(k r)^{2}}-\frac{3 \cos k r}{(k r)^{3}}+\frac{3 \sin k r}{(k r)^{4}} \\
& \operatorname{Im}\left[F_{\|}(r)\right]=\frac{\cos k r}{(k r)^{2}}-\frac{3 \sin k r}{(k r)^{3}}-\frac{3 \cos k r}{(k r)^{4}}, \\
& \operatorname{Re}\left[F_{\perp}(r)\right]=-\frac{\cos k r}{k r}+\frac{3 \sin k r}{(k r)^{2}}+\frac{6 \cos k r}{(k r)^{3}}-\frac{6 \sin k r}{(k r)^{4}}
\end{aligned}
$$

and

$$
\operatorname{Im}\left[F_{\perp}(r)\right]=-\frac{\sin k r}{k r}-\frac{3 \cos k r}{(k r)^{2}}+\frac{6 \sin k r}{(k r)^{3}}+\frac{6 \cos k r}{(k r)^{4}} .
$$

Having chosen a basic quadrupole, or a combination of these to represent the specific quadrupole of a particular problem, the final step of the calculation is the implementation of Eq. (47); the combination of the dipole moment components Eq. (48) with the fields of Table A2. If, for example, we have an XY quadrupole then Eq. (47) becomes: 


$$
\begin{aligned}
W= & -3 K F_{\|}(r) \sin ^{2} \vartheta \sin 2 \varphi\left[\sin \vartheta \cos \varphi \mu_{x}+\sin \vartheta \sin \varphi \mu_{y}+\cos \vartheta \mu_{z}\right] \\
& -K F_{\perp}(r) \sin \vartheta \cos \vartheta \sin 2 \varphi\left[\cos \vartheta \cos \varphi \mu_{x}+\cos \vartheta \sin \varphi \mu_{y}-\sin \vartheta \mu_{z}\right] \\
& -K F_{\perp}(r) \sin \vartheta \cos 2 \varphi\left[-\sin \varphi \mu_{x}+\cos \varphi \mu_{y}\right]
\end{aligned}
$$

The second method we employ is based upon the Eq. (45), given by Jackson [61] for the energy, $W$, of a quadrupole, $Q_{\alpha \beta}$, in the gradient of the field of a dipole, $E^{D}$. In the interest of brevity we confine our attention to the near zone $(k R \ll 1)$ which, as Scholes and Andrews [67] have pointed out, is the region in which higher multipole interactions are most likely to be significant. We calculate the elements of the quadrupole tensor using Eq. (32) [16], but it should be noted that, because expression (8) for the quadrupole moment is onesixth of that used by Jackson [61], the factor of 1/6 in his expression is omitted, i.e.

$$
W=-\sum_{\alpha} \sum_{\beta} Q_{\alpha \beta} \frac{\partial E_{\alpha}^{D}}{\partial \beta} .
$$

For an out-going wave, the Cartesian components of the electric field of the dipole are related to the polar components in the following manner:

$\widehat{\vec{E}}_{x}=\cos \vartheta \cos \varphi \hat{\vec{E}}_{\Theta}-\sin \varphi \hat{\vec{E}}_{\Phi}+\sin \vartheta \cos \varphi \hat{\vec{E}}_{R}$

$\widehat{\vec{E}}_{y}=\cos \vartheta \sin \varphi \hat{\vec{E}}_{\Theta}+\cos \varphi \hat{\vec{E}}_{\Phi}+\sin \vartheta \sin \varphi \hat{\vec{E}}_{R}$

$\widehat{\vec{E}}_{z}=-\sin \vartheta \hat{\vec{E}}_{\Theta}+\cos \vartheta \hat{\vec{E}}_{R}$

And the three Cartesian components of a dipole, $\vec{\mu}$, generate an electric field with components along the polar vectors as shown in the Table A3.

Thus, 


$$
\begin{gathered}
\vec{E}_{x}=\cos \vartheta \cos \varphi \vec{E}_{\theta}-\sin \varphi \vec{E}_{\Phi}+\sin \vartheta \cos \varphi \vec{E}_{R} \\
\vec{E}_{x}=\frac{1}{4 \pi \varepsilon_{o} R^{3}}\left\{\cos \vartheta \cos \varphi\left[-\mu_{x} \cos \vartheta \cos \varphi-\mu_{y} \cos \vartheta \sin \varphi+\mu_{z} \sin \vartheta\right]\right. \\
\left.-\sin \varphi\left[\mu_{x} \sin \varphi-\mu_{y} \cos \varphi\right]+2 \sin \vartheta \cos \varphi\left[\mu_{x} \sin \vartheta \cos \varphi+\mu_{y} \sin \vartheta \sin \varphi+\mu_{z} \cos \vartheta\right]\right\} \\
=\frac{1}{4 \pi \varepsilon_{o} R^{3}}\left\{\mu_{x}\left[3 \sin ^{2} \vartheta \cos ^{2} \varphi-1\right]+3 \mu_{y} \sin ^{2} \vartheta \sin \varphi \cos \varphi+3 \mu_{z} \sin \vartheta \cos \vartheta \cos \varphi\right\},
\end{gathered}
$$

In the same manner we can obtain:

$$
\vec{E}_{y}=\frac{1}{4 \pi \varepsilon_{o} R^{3}}\left\{3 \mu_{x} \sin ^{2} \vartheta \sin \varphi \cos \varphi+\mu_{y}\left[3 \sin ^{2} \vartheta \sin ^{2} \varphi-1\right]+3 \mu_{z} \sin \vartheta \cos \vartheta \sin \varphi\right\}
$$

and

$$
\vec{E}_{z}=\frac{1}{4 \pi \varepsilon_{o} R^{3}}\left\{3 \mu_{x} \sin \vartheta \cos \vartheta \cos \varphi+3 \mu_{y} \sin \vartheta \cos \vartheta \sin \varphi+\mu_{z}\left[3 \cos ^{2} \vartheta-1\right]\right\} .
$$

We now have to convert to Cartesian co-ordinateśs because Jackson's formula depends upon a Taylor expansion in Cartesian co-ordinates. Carrying out the conversion and differentiating with resect to the Cartesian co-ordinates we obtain the contributions of the components of the dipole to the derivatives of the fields, as shown in Table A4. Converting back to spherical polar co-ordinates the derivatives can also be obtained, as shown in Table A5.

On p. 5382 of their paper, Scholes and Andrews [69] give expressions for the multipole couplings in the near zone where the QED equations asymptote to the classical results. They also show how orientation factors for the couplings may be obtained in their PW treatment. In the following we compare their results for the near zone with those deduced by the procedures described above. As far as the dipole-quadrupole coupling is concerned, we are interested in the equation:

$$
M_{d a}^{\text {Coul }}=\left(\mu_{\alpha}^{0 n} Q_{\beta \gamma}^{m 0}-Q_{\beta \gamma}^{0 n} \mu_{\alpha}^{m 0}\right) V_{\alpha \beta \gamma}
$$


Where, in the near zone, the interaction tensor, $V_{\alpha \beta \gamma}$, becomes:

$$
V_{\alpha \beta \gamma}=\frac{1}{4 \pi \varepsilon_{o} R^{4}}\left[15 \hat{\vec{R}}_{\alpha} \hat{\vec{R}}_{\beta} \hat{\vec{R}}_{\gamma}-3\left(\delta_{\alpha \beta} \hat{\vec{R}}_{\gamma}+\delta_{\alpha \gamma} \hat{\vec{R}}_{\beta}+\delta_{\beta \gamma} \hat{\vec{R}}_{\alpha}\right)\right]
$$

Orientation factors are obtained by making the substitutions:

$$
\delta_{\alpha \beta} \rightarrow \hat{\vec{\alpha}} \cdot \hat{\vec{\beta}} \text { and } \hat{\vec{R}}_{\alpha} \rightarrow \hat{\vec{\alpha}} \cdot \hat{\vec{R}} \text {, }
$$

where $\hat{\vec{\alpha}}$ denotes a unit vector in the direction of the tensor component $\alpha$ of the transition moment and $\vec{R}$ is the centre-to-centre vector. We compare the formula for $V_{\alpha \beta \gamma}$ from [69] with Table A4 in which the substitution $\mathrm{R}^{2}=\mathrm{x}^{2}+\mathrm{y}^{2}+\mathrm{z}^{2}$ has been made, as is shown in Table A6. Each entry in the table describes the interaction, through the dipole-field gradient, between the component of the dipole at the head of the column with the quadrupole component at the beginning of each row. We can see that there are only three types of entry for $\mu_{\alpha}^{0 n} Q_{\beta \gamma}^{m 0}$.

$$
V_{\alpha \beta \gamma}=\frac{1}{4 \pi \varepsilon_{o} R^{4}}\left[15 \hat{\vec{R}}_{\alpha} \hat{\vec{R}}_{\beta} \hat{\vec{R}}_{\gamma}-3\left(\delta_{\alpha \beta} \hat{\vec{R}}_{\gamma}+\delta_{\alpha \gamma} \hat{\vec{R}}_{\beta}+\delta_{\beta \gamma} \hat{\vec{R}}_{\alpha}\right)\right]
$$

Case 1. $\alpha=\beta=\gamma$. For example, in the case where $\alpha=\beta=\gamma=x$.

$$
\begin{aligned}
V_{x x x} & =\frac{1}{4 \pi \varepsilon_{o} R^{4}}\left[15 \hat{\vec{R}}_{x} \hat{\vec{R}}_{x} \hat{\vec{R}}_{x}-9 \delta_{x x} \hat{\vec{R}}_{x}\right]=\frac{1}{4 \pi \varepsilon_{o} R^{4}}\left[15(\hat{\vec{x}} \cdot \hat{\vec{R}})^{3}-9(\hat{\vec{x}} \cdot \hat{\vec{x}})(\hat{\vec{x}} \cdot \hat{\vec{R}})\right] \\
& =\frac{3}{4 \pi \varepsilon_{o} R^{4}}\left[5(\hat{\vec{x}} \cdot \hat{\vec{R}})^{3}-3(\hat{\vec{x}} \cdot \hat{\vec{R}})\right]=\frac{3}{4 \pi \varepsilon_{o} R^{4}}\left[5\left(\frac{\hat{\vec{x}} \cdot \vec{R}}{R}\right)^{3}-3\left(\frac{\hat{\vec{x}} \cdot \vec{R}}{R}\right)\right] \\
& =\frac{3}{4 \pi \varepsilon_{o} R^{7}}\left[5(\hat{\vec{x}} \cdot \vec{R})^{3}-3 R^{2}(\hat{\vec{x}} \cdot \vec{R})\right]=\frac{3(\hat{\vec{x}} \cdot \vec{R})}{4 \pi \varepsilon_{o} R^{7}}\left[5(\hat{\vec{x}} \cdot \vec{R})^{2}-3 R^{2}\right]
\end{aligned}
$$

But, $\hat{\vec{x}} \cdot \vec{R}=x$ and we can therefore write: 


$$
V_{x x x}=\frac{3}{4 \pi \varepsilon_{o} R^{7}} x\left[5 x^{2}-3 R^{2}\right]
$$

which is the negative of the top-left entry in Table A6. The negative sign is no problem, because it simply reflects the relative orientations of the dipole and the quadrupole.

Case 2. $\alpha=\beta$ only. For example, for the case where $\alpha=\beta=x, \gamma=y$.

$$
\begin{gathered}
V_{x x y}=\frac{1}{4 \pi \varepsilon_{o} R^{4}}\left[15 \hat{\vec{R}}_{x} \hat{\vec{R}}_{x} \hat{\vec{R}}_{y}-3\left(\delta_{x x} \hat{\vec{R}}_{y}+\delta_{x y} \hat{\vec{R}}_{x}+\delta_{x y} \hat{\vec{R}}_{x}\right)\right] \\
=\frac{3}{4 \pi \varepsilon_{o} R^{4}}\left[5(\hat{\vec{x}} \cdot \hat{\vec{R}})^{2}(\hat{\vec{y}} \cdot \hat{\vec{R}})-(\hat{\vec{x}} \cdot \hat{\vec{x}})(\hat{\vec{y}} \cdot \hat{\vec{R}})\right] \\
=\frac{3}{4 \pi \varepsilon_{o} R^{4}}\left[5\left(\frac{\hat{\vec{x}} \cdot \vec{R}}{R}\right)^{2}\left(\frac{\hat{\vec{y}} \cdot \vec{R}}{R}\right)-\left(\frac{\hat{\vec{y}} \cdot \vec{R}}{R}\right)\right]=\frac{3}{4 \pi \varepsilon_{o} R^{7}}\left[5(\hat{\vec{x}} \cdot \vec{R})^{2}(\hat{\vec{y}} \cdot \vec{R})-R^{2}(\hat{\vec{y}} \cdot \vec{R})\right]
\end{gathered}
$$

and we have:

$$
V_{x x y}=\frac{3}{4 \pi \varepsilon_{o} R^{7}} y\left[5 x^{2}-R^{2}\right]
$$

Case 3. $\quad \alpha, \beta$ and $\gamma$ all different. For example, $\alpha=x, \beta=y$ and $\gamma=z$.

$$
V_{x y z}=\frac{1}{4 \pi \varepsilon_{o} R^{4}}\left[15 \hat{\vec{R}}_{x} \hat{\vec{R}}_{y} \widehat{\hat{\vec{R}}}_{z}\right]=\frac{3}{4 \pi \varepsilon_{o} R^{4}}[5(\hat{\vec{x}} \cdot \hat{\vec{R}})(\hat{\vec{y}} \cdot \hat{\vec{R}})(\hat{\vec{z}} \cdot \hat{\vec{R}})]=\frac{3}{4 \pi \varepsilon_{o} R^{7}}[5 x y z]
$$

Which agrees exactly with the PW results of the Scholes and Andrews for dipole-quadrupole interaction.

\section{Group Theory and Spherical Symmetry}

\subsection{The symmetry species of the $\mid J l M>$}

There are few, if any, examples of the application of the VSH description of the interaction of light with matter to molecules. The d-d transitions of transition metal ion 
complexes and the f-f and f-d transitions of the lanthanides [76, 77] have been widely studied and they provide very good examples of the way in which the scope of the VSH calculus can be augmented by combination with point-group theory. The development follows the ligand field theory very closely $[78,79]$. We note that there are some recent studies investigating higher order multipole effects in these systems [80, 81]

The symmetry of the SSHs, $\mid l m>$, which we encounter in atomic orbital functions are determined by the quantum numbers $l$ and $m$ [82]. They are widely tabulated [83]. Similarly, the symmetries of the VSHs, $|J l M\rangle$, are determined by $J$ and $M$ [Va] and SSH tables can be used directly for the VSHs. The parity, $\pi$, of $|l m\rangle$ is determined by $l, \pi|l m\rangle=$ $(-1)^{l} \mid l m>$, and for VSHs, $\mid J l M>(l=J, J \pm 1)$, formed from unit vectors, $\hat{\vec{e}}_{1 n}$, having axial symmetry, $\pi\left|J l M>=(-1)^{l}\right| J l M>$ also.

The selection rules which determine the values of $J, l$ and $M$ in $Y_{J I M}$ for a non-zero value of the matrix element $\left\langle Y_{G H}\left|\boldsymbol{Y}_{J I M} \nabla\right| Y_{A C}\right\rangle$ are [49] \{We use the alternative symbol, $\boldsymbol{Y}_{J I M} \equiv|J l M\rangle$, here to avoid problems with notation.\}:
1) $|G-A| \leq J \leq(G+A)$
2) $M=H-C$ with $|M| \leq J$
3) $l+A+G$ must be odd.

As examples, the symmetry species of these matrix elements for the possible d-d, f-f and f$\mathrm{d}$ transitions of metal ions in a variety of environments are shown in Tables B1-B6. We tabulate the symmetry species of $Y_{J I M}, \nabla$ and their scalar product, $Y_{J l M} \bullet \nabla$.

Selection rules for $\mathrm{d}-\mathrm{d}$ transitions; $\boldsymbol{Y}_{J l M} .1 \leq J \leq 4,|M| \leq J, l$ must be odd.

Table A.1 includes the five transitions between two identical d orbitals. They can be eliminated by removing the value for $\mu=0$ from each column. However, such transitions are not unrealisable [84]: though they may not contribute to the intensity, one can envisage a transition between two molecular orbitals each of which contains a contribution from the same d-orbital.

\subsection{The symmetries of electric and magnetic fields}

Electric fields are characterised by a single value of $J$ and $M$ and two values of $l$ [44]. Since the values of $l$ differ by 2 , the two contributions to the field have the same parity $(-1)^{l}$, as must obviously be the case. Magnetic fields are characterised by single values of $J, M$ and $l$ [44]. Thus, particular symmetry species may be assigned to electric and magnetic fields generated in molecular environments and the well-known methods of group theory can be 
applied to the determination of, for example, the combination of the dipoles of surrounding molecules or ligands with which a central field can interact.

\subsection{Advanced use of point-group theory}

It was recognised in the early 1960's, especially by Griffith [83] and by Koster et al. [86] building on the work of Wigner [87] and Racah [88, 89, 90], that the concepts relating to the coupling of angular momenta, well-known under spherical symmetry ( $\left.\mathrm{SO}_{3}\right)$, could be extended to the irreducible representations of the point groups of lower symmetry. These methods, sometimes known as Wigner-Racah calculus, have been developed, explored thoroughly and systematized from the mathematical standpoint, by Butler [91, 92]. His particular and precise approach has been exploited and illustrated with many examples by Piepho and Schatz [93], who have shown how remarkably powerful these methods can be in the solution of problems in molecular spectroscopy. Thus, the tables above can be used as they stand for comparatively simple problems and they also form the basis upon which more advanced calculations can be performed. Natural circular dichroism provides an example of the former.

As is well-known [16], circular dichroism, the differential absorption of left and right circularly polarised light, is seen in the transition $m \leftarrow 0$ when the scalar product of the electric and magnetic dipole transition moments of $\mathrm{m} \leftarrow 0$ has a finite value. The magnetic moment is characterised by the VSH $\boldsymbol{Y}_{11 \mathrm{M}}$ and the electric moment by $\sqrt{ }(2 / 3) \boldsymbol{Y}_{10 \mathrm{M}}+$ $\sqrt{ }(1 / 3) \boldsymbol{Y}_{12 \mathrm{M}}[34]$, and these VSHs will belong to the same irreducible representations of any symmetry point group because this depends only upon $J$. However, their parities, determined by $(-1)^{l}$, are $\mathrm{u}$ and $\mathrm{g}$ respectively. Accordingly, in any environment in which a distinction between $\mathrm{u}$ and $\mathrm{g}$ can be made, i.e. where a plane or centre of symmetry exist, there can be no circular dichroism. In systems which lack these symmetry elements, $C_{n}, D_{n}$, $T, O$ and $I(\mathrm{n}>2)$ circular dichroism may be observed. For the sake of completeness we add the additional requirement that an optically active molecule must also be devoid of an alternating rotation-rêflexion/axis of symmetry [94], though this has no bearing upon the present discussion.

\section{Outlook}

We begin with an important generalization. The intrinsic separation of radial and angular terms in an SW calculation has two significant and valuable consequences. Firstly, the 
angular terms in any calculation are unchanged from those of the corresponding classical electrodynamical theory and full use of well-established classical results can be made. Secondly, all the effects of retardation are rigorously confined to the radial terms which makes the calculation and the formulation of the results simpler. These points are illustrated well by the SW calculation of the dipole-dipole interaction energy and energy-transfer, recently described in detail [73], where the SW approach removes completely the requirement for a problematic integration over wave vectors and shows clearly the origin of the ambiguous sign in the imaginary contribution to the coupling.

In the case of selection rules in atomic spectroscopy the following might also be added to the points made above. Whereas in the PW description E2 and M1 transitions arise together as the second term in the expansion of the exponential, they are completely distinct in the SW description since the parameters which define them differ in the value of $l ; J=2, l=1$ and 3 for E2 and $J=2, l=2$ for M1 [49].

With regard to the description of emitted radiation the first point to be noted is the generality of the PW approach, in this particular aspect of its application, where it is only necessary to specify the multipolarity of the source, dipole, quadrupole, .... This degree of generality is not readily possible with the SW method in which it is expedient to consider the emission from a defined atomic-spectral process. This fixes the quantum number parameters, $J, l$ and $M$, of the description of the radiation in terms of the product of a radial function, a Hankel function of the first kind $h_{l}^{(1)}(k r)$, and an angular function, a vector spherical harmonic $\mid \operatorname{Jl} M(\vartheta, \varphi)>[42]$, which describes not only the angular distribution of the emerging photon, in terms of the polar coordinates $\vartheta$ and $\varphi$, but also, through $\widehat{\vec{\Theta}}$ and $\widehat{\vec{\Phi}}$, its polarization, i.e. the orientation of the associated electric and magnetic fields. Thus, the SW technique, while lacking in generality, gives more information about the functional form of the emitted radiation, which can be written down immediately once the parameters $J, l$ and $M$ have been fixed. And this is true for any degree of multipolarity so that the level of extra work and complexity increase only slowly with increasing $J$, though a second calculation is required if electric and magnetic field strengths as a function of a specified multipole moment are required. Furthermore, since the combined functions, $\operatorname{Jl} M(\vartheta, \varphi)>\times h_{l}{ }^{(1)}(k r)$ are solutions of Maxwell's equations they are fully retarded. But the reliance on spherical symmetry is an important limitation to application and suggests a reason for the complete absence of application in molecular, though not in atomic spectroscopy. Nevertheless, preliminary investigations, some of them outlined in Section 5 above, demonstrate that the 
group-theoretical algebra, which has been applied so successfully to molecular spectroscopy, especially to the ligand field theory of transition metal ions [83, 91], can be similarly used with the angular functions, $\mid \operatorname{Jl} M(\vartheta, \varphi)>$, offering a significant broadening of the SW method into important new areas of application.

Perhaps one of the important advantages of the SW description, over that of the PW description is the way longitudinal and transverse field components naturally arise in the SW description. The SW approach effectively eliminates the need to define an external coordinate system, with the emitting multipole defining a natural origin. Future work may involve attempting to unify the SW approach to models for structured light, with the aim of studying angular momentum transfer, a subject that has been recently considered by the authors [95].

\section{Acknowledgements}

We'd like to thank David Hughes for help with the crystallography of anthracene.

\section{Appendix A: Tables relating to dipole and quadrupole interactions}

Table A1. Quadrupole moments calculated using equation 10

\begin{tabular}{|l|c|c|c|c|c|c|}
\hline & $\mathrm{Q}_{\mathrm{xx}}$ & $\mathrm{Q}_{\mathrm{yy}}$ & $\mathrm{Q}_{\mathrm{zz}}$ & $\mathrm{Q}_{\mathrm{xy}}=\mathrm{Q}_{\mathrm{yx}}$ & $\mathrm{Q}_{\mathrm{xz}}=\mathrm{Q}_{\mathrm{zx}}$ & $\mathrm{Q}_{\mathrm{yz}}=\mathrm{Q}_{\mathrm{zy}}$ \\
\hline $\mathrm{XY}$ & & & & $s^{2} q$ & & \\
\hline $\mathrm{XZ}$ & & & & & $s^{2} q$ & \\
\hline $\mathrm{YZ}$ & & & & & & $s^{2} q$ \\
\hline $\mathrm{X}^{2}$ & $s^{2} q$ & & & & & \\
\hline $\mathrm{Y}^{2}$ & & $s^{2} q$ & & & & \\
\hline $\mathrm{Z}^{2}$ & & & $s^{2} q$ & & & \\
\hline
\end{tabular}

Table A2. Quadrupole fields 


\begin{tabular}{|l|c|c|c|}
\hline & $\vec{E}_{\Theta}$ & $\vec{E}_{\Phi}$ & $\vec{E}_{R}$ \\
\hline $\mathrm{XY}$ & $K F_{\Theta}(r) \sin \vartheta \cos \vartheta \sin 2 \varphi \vec{\Theta}$ & $K F_{\Phi}(r) \sin \vartheta \cos 2 \varphi \vec{\Phi}$ & $3 K F_{R}(r) \sin ^{2} \vartheta \sin 2 \varphi \vec{R}$ \\
\hline $\mathrm{XZ}$ & $K F_{\Theta}(r) \cos 2 \vartheta \cos \varphi \vec{\Theta}$ & $-K F_{\Phi}(r) \cos \vartheta \sin \varphi \vec{\Phi}$ & $3 K F_{R}(r) \sin 2 \vartheta \cos \varphi \vec{R}$ \\
\hline $\mathrm{YZ}$ & $K F_{\Theta}(r) \cos 2 \vartheta \sin \varphi \vec{\Theta}$ & $K F_{\Phi}(r) \cos \vartheta \cos \varphi \vec{\Phi}$ & $3 K F_{R}(r) \sin 2 \vartheta \sin \varphi \vec{R}$. \\
\hline $\mathrm{X}^{2}$ & $2 K F(r) \sin \vartheta \cos \vartheta \cos { }^{2} \overrightarrow{ }$ & $-2 K F(r) \sin \vartheta \sin \varphi \cos \varphi$ & \\
\hline $\mathrm{Y}^{2}$ & $2 K F(r) \sin \vartheta \cos \vartheta \sin ^{2} \varphi$ & $2 K F(r) \sin \vartheta \sin \varphi \cos \varphi \vec{r}$ & {$\left[3 \sin ^{2} \vartheta \cos ^{2} \varphi-1\right] \vec{R}$} \\
& $-2 K F(r) \sin \vartheta \cos \vartheta$ & & {$\left[3 \sin ^{2} \vartheta \sin ^{2} \varphi-1\right] \vec{R}$} \\
\hline $\mathrm{Z}^{2}$ & & 0 & $2 K F_{R}(r)\left[3 \cos ^{2} \vartheta-1\right] \vec{R}$ \\
\hline
\end{tabular}

Where

$$
K=\frac{i q s^{2} k^{4}}{8 \pi \varepsilon_{o}} \equiv \frac{i Q k^{4}}{8 \pi \varepsilon_{o}}
$$

Table A3. Dipole field components in spherical polar co-ordinates

\begin{tabular}{|c|r|r|r|}
\hline Dipole, $\mu$, along & $\vec{E}_{\Theta}$ & $\vec{E}$ & $\vec{E}_{R}$ \\
\hline $\mathrm{X}$ & $-\frac{\mu \cos \vartheta \cos \varphi}{4 \pi \varepsilon_{o} R^{3}}$ & $+\frac{\mu \sin \varphi}{4 \pi \varepsilon_{o} R^{3}}$ & $+\frac{\mu \sin \vartheta \cos \varphi}{2 \pi \varepsilon_{o} R^{3}}$ \\
\hline $\mathrm{Y}$ & $-\frac{\mu \cos \vartheta \sin \varphi}{4 \pi \varepsilon_{o} R^{3}}$ & $-\frac{\mu \cos \varphi}{4 \pi \varepsilon_{o} R^{3}}$ & $+\frac{\mu \sin \vartheta \sin \varphi}{2 \pi \varepsilon_{o} R^{3}}$ \\
\hline $\mathrm{Z}$ & $+\frac{\mu \sin \vartheta}{4 \pi \varepsilon_{o} R^{3}}$ & 0 & $+\frac{\mu \cos \vartheta}{2 \pi \varepsilon_{o} R^{3}}$ \\
\hline
\end{tabular}

Table A4. Dipole field derivatives in Cartesian co-ordinates. Each cell is to be multiplied by $3 / 4 \pi \varepsilon_{0} R^{7}$

\begin{tabular}{|l|l|c|}
\hline$\mu_{\mathrm{x}}$ & $\mu_{\mathrm{y}}$ & $\mu_{\mathrm{z}}$ \\
\hline
\end{tabular}




\begin{tabular}{|c|c|c|c|}
\hline$\partial \mathrm{E}_{\mathrm{x}} / \partial \mathrm{x}$ & $\mathrm{x}\left[-2 \mathrm{x}^{2}+3 \mathrm{y}^{2}+3 \mathrm{z}^{2}\right]$ & $\mathrm{y}\left[-4 \mathrm{x}^{2}+\mathrm{y}^{2}+\mathrm{z}^{2}\right]$ & $\mathrm{z}\left[-4 \mathrm{x}^{2}+\mathrm{y}^{2}+\mathrm{z}^{2}\right]$ \\
\hline$\partial \mathrm{E}_{\mathrm{y}} / \partial \mathrm{y}$ & $\mathrm{x}\left[\mathrm{x}^{2}-4 \mathrm{y}^{2}+\mathrm{z}^{2}\right]$ & $\mathrm{y}\left[3 \mathrm{x}^{2}-2 \mathrm{y}^{2}+3 \mathrm{z}^{2}\right]$ & $\mathrm{z}\left[\mathrm{x}^{2}-4 \mathrm{y}^{2}+\mathrm{z}^{2}\right]$ \\
\hline$\partial \mathrm{E}_{\mathrm{z}} / \partial \mathrm{z}$ & $\mathrm{x}\left[\mathrm{x}^{2}+\mathrm{y}^{2}-4 \mathrm{z}^{2}\right]$ & $\mathrm{y}\left[\mathrm{x}^{2}+\mathrm{y}^{2}-4 \mathrm{z}^{2}\right]$ & $\mathrm{z}\left[3 \mathrm{x}^{2}+3 \mathrm{y}^{2}-2 \mathrm{z}^{2}\right]$ \\
\hline$\partial \mathrm{E}_{\mathrm{x}} / \partial \mathrm{y}$ & $\mathrm{y}\left[-4 \mathrm{x}^{2}+\mathrm{y}^{2}+\mathrm{z}^{2}\right]$ & $\mathrm{x}\left[\mathrm{x}^{2}-4 \mathrm{y}^{2}+\mathrm{z}^{2}\right]$ & $-5 \mathrm{xyz}$ \\
\hline$\partial \mathrm{E}_{\mathrm{x}} / \partial \mathrm{z}$ & $\mathrm{z}\left[-4 \mathrm{x}^{2}+\mathrm{y}^{2}+\mathrm{z}^{2}\right]$ & $-5 \mathrm{xyz}$ & $\mathrm{x}\left[\mathrm{x}^{2}+\mathrm{y}^{2}-4 \mathrm{z}^{2}\right]$ \\
\hline$\partial \mathrm{E}_{\mathrm{y}} / \partial \mathrm{x}$ & $\mathrm{y}\left[-4 \mathrm{x}^{2}+\mathrm{y}^{2}+\mathrm{z}^{2}\right]$ & $\mathrm{x}\left[\mathrm{x}^{2}-4 \mathrm{y}^{2}+\mathrm{z}^{2}\right]$ & $-5 \mathrm{xyz}$ \\
\hline$\partial \mathrm{E}_{\mathrm{y}} / \partial \mathrm{z}$ & $-5 \mathrm{xyz}$ & $\mathrm{z}\left[\mathrm{x}^{2}-4 \mathrm{y}^{2}+\mathrm{z}^{2}\right]$ & $\mathrm{y}\left[\mathrm{x}^{2}+\mathrm{y}^{2}-4 \mathrm{z}^{2}\right]$ \\
\hline$\partial \mathrm{E}_{\mathrm{z}} / \partial \mathrm{x}$ & $\mathrm{z}\left[-4 \mathrm{x}^{2}+\mathrm{y}^{2}+\mathrm{z}^{2}\right]$ & $-5 \mathrm{xyz}$ & $\mathrm{x}\left[\mathrm{x}^{2}+\mathrm{y}^{2}-4 \mathrm{z}^{2}\right]$ \\
\hline$\partial \mathrm{E}_{\mathrm{z}} / \partial \mathrm{y}$ & $-5 \mathrm{xyz}$ & $\mathrm{z}\left[\mathrm{x}^{2}-4 \mathrm{y}^{2}+\mathrm{z}^{2}\right]$ & $\mathrm{y}\left[\mathrm{x}^{2}+\mathrm{y}^{2}-4 \mathrm{z}^{2}\right]$ \\
\hline
\end{tabular}

Table A5. Dipole field derivatives in spherical polar co-ordinates. Each cell is to be multiplied by $3 / 4 \pi \varepsilon_{0} R^{4}$

\begin{tabular}{|l|c|c|c|}
\hline & $\mu_{\mathrm{x}}$ & $\mu_{\mathrm{y}}$ & $\mu_{\mathrm{z}}$ \\
\hline$\partial \mathrm{E}_{\mathrm{x}} / \partial \mathrm{x}$ & $\sin \vartheta \cos \phi\left[3-5 \sin ^{2} \vartheta \cos ^{2} \phi\right]$ & $\sin \vartheta \sin \phi\left[1-5 \sin ^{2} \vartheta \cos ^{2} \phi\right]$ & $\cos \vartheta\left[1-5 \sin ^{2} \vartheta \cos ^{2} \phi\right]$ \\
\hline$\partial \mathrm{E}_{\mathrm{y}} / \partial \mathrm{y}$ & $\sin \vartheta \cos \phi\left[1-5 \sin ^{2} \vartheta \sin ^{2} \phi\right]$ & $\sin \vartheta \sin \phi\left[3-5 \sin ^{2} \vartheta \sin ^{2} \phi\right]$ & $\cos \vartheta\left[1-5 \sin ^{2} \vartheta \sin ^{2} \phi\right]$ \\
\hline$\partial \mathrm{E}_{\mathrm{z}} / \partial \mathrm{z}$ & $\sin \vartheta \cos \phi\left[1-5 \cos ^{2} \vartheta\right]$ & $\sin \vartheta \sin \phi\left[1-5 \cos ^{2} \vartheta\right]$ & $\cos \vartheta\left[3-5 \cos ^{2} \vartheta\right]$ \\
\hline$\partial \mathrm{E}_{\mathrm{x}} / \partial \mathrm{y}$ & $\sin \vartheta \sin \phi\left[1-5 \sin ^{2} \vartheta \cos ^{2} \phi\right]$ & $\sin \vartheta \cos \phi\left[1-5 \sin ^{2} \vartheta \sin ^{2} \phi\right]$ & $-5 \sin ^{2} \vartheta \cos \vartheta \sin \phi \cos \phi$ \\
\hline$\partial \mathrm{E}_{\mathrm{x}} / \partial \mathrm{z}$ & $\cos \vartheta\left[1-5 \sin ^{2} \vartheta \cos ^{2} \phi\right]$ & $-5 \sin ^{2} \vartheta \cos \vartheta \sin \phi \cos \phi$ & $\sin \vartheta \cos \phi\left[1-5 \cos ^{2} \vartheta\right]$ \\
\hline$\partial \mathrm{E}_{\mathrm{y}} / \partial \mathrm{x}$ & $\sin \vartheta \sin \phi\left[1-5 \sin ^{2} \vartheta \cos ^{2} \phi\right]$ & $\sin \vartheta \cos \phi\left[1-5 \sin ^{2} \vartheta \sin ^{2} \phi\right]$ & $-5 \sin { }^{2} \vartheta \cos \vartheta \sin \phi \cos \phi$ \\
\hline$\partial \mathrm{E}_{\mathrm{y}} / \partial \mathrm{z}$ & $-5 \sin { }^{2} \vartheta \cos \vartheta \sin \phi \cos \phi$ & $\cos \vartheta\left[1-5 \sin ^{2} \vartheta \sin ^{2} \phi\right]$ & $\sin \vartheta \sin \phi\left[1-5 \cos ^{2} \vartheta\right]$ \\
\hline$\partial \mathrm{E}_{\mathrm{z}} / \partial \mathrm{x}$ & $\cos \vartheta\left[1-5 \sin ^{2} \vartheta \cos ^{2} \phi\right]$ & $-5 \sin { }^{2} \vartheta \cos \vartheta \sin ^{2} \phi \cos ^{2} \phi$ & $\sin \vartheta \cos \phi\left[1-5 \cos ^{2} \vartheta\right]$ \\
\hline$\partial \mathrm{E}_{\mathrm{z}} / \partial \mathrm{y}$ & $-5 \sin ^{2} \vartheta \cos \vartheta \sin \phi \cos \phi$ & $\cos \vartheta\left[1-5 \sin ^{2} \vartheta \sin ^{2} \phi\right]$ & $\sin \vartheta \sin \phi\left[1-5 \cos ^{2} \vartheta\right]$ \\
\hline
\end{tabular}

Table A6. Quadrupole field gradients in Cartesian co-ordinates. Each cell is to be multiplied by $3 / 4 \pi \varepsilon_{\mathrm{o}} r^{7}$.

\begin{tabular}{|c|l|l|l|c|}
\hline $\mathrm{Q}_{\beta \gamma}$ & Gradient & $\mu_{\mathrm{x}}$ & $\mu_{\mathrm{y}}$ & $\mu_{\mathrm{z}}$ \\
\hline $\mathrm{Q}_{\mathrm{xx}}$ & $\partial \mathrm{E}_{\mathrm{x}} / \partial \mathrm{x}$ & $\mathrm{x}\left[3 \mathrm{R}^{2}-5 \mathrm{x}^{2}\right]$ & $\mathrm{y}\left[\mathrm{R}^{2}-5 \mathrm{x}^{2}\right]$ & $\mathrm{z}\left[\mathrm{R}^{2}-5 \mathrm{x}^{2}\right]$ \\
\hline $\mathrm{Q}_{\mathrm{yy}}$ & $\partial \mathrm{E}_{\mathrm{y}} / \partial \mathrm{y}$ & $\mathrm{x}\left[\mathrm{R}^{2}-5 \mathrm{y}^{2}\right]$ & $\mathrm{y}\left[3 \mathrm{R}^{2}-5 \mathrm{y}^{2}\right]$ & $\mathrm{z}\left[\mathrm{R}^{2}-5 \mathrm{y}^{2}\right]$ \\
\hline $\mathrm{Q}_{\mathrm{zz}}$ & $\partial \mathrm{E}_{\mathrm{z}} / \partial \mathrm{z}$ & $\mathrm{x}\left[\mathrm{R}^{2}-5 \mathrm{z}^{2}\right]$ & $\mathrm{y}\left[\mathrm{r}^{2}-5 \mathrm{z}^{2}\right]$ & $\mathrm{z}\left[3 \mathrm{R}^{2}-5 \mathrm{z}^{2}\right]$ \\
\hline $\mathrm{Q}_{\mathrm{yx}}$ & $\partial \mathrm{E}_{\mathrm{x}} / \partial \mathrm{y}$ & $\mathrm{y}\left[\mathrm{R}^{2}-5 \mathrm{x}^{2}\right]$ & $\mathrm{x}\left[\mathrm{R}^{2}-5 \mathrm{y}^{2}\right]$ & $-5 \mathrm{xyz}$ \\
\hline
\end{tabular}




\begin{tabular}{|c|c|c|c|c|}
\hline $\mathrm{Q}_{\mathrm{zx}}$ & $\partial \mathrm{E}_{\mathrm{x}} / \partial \mathrm{z}$ & $\mathrm{z}\left[\mathrm{R}^{2}-5 \mathrm{x}^{2}\right]$ & $-5 \mathrm{xyz}$ & $\mathrm{x}\left[\mathrm{R}^{2}-5 \mathrm{z}^{2}\right]$ \\
\hline $\mathrm{Q}_{\mathrm{xy}}$ & $\partial \mathrm{E}_{\mathrm{y}} / \partial \mathrm{x}$ & $\mathrm{y}\left[\mathrm{R}^{2}-5 \mathrm{x}^{2}\right]$ & $\mathrm{x}\left[\mathrm{R}^{2}-5 \mathrm{y}^{2}\right]$ & $-5 \mathrm{xyz}$ \\
\hline $\mathrm{Q}_{\mathrm{zy}}$ & $\partial \mathrm{E}_{\mathrm{y}} / \partial \mathrm{z}$ & $-5 \mathrm{xyz}$ & $\mathrm{z}\left[\mathrm{R}^{2}-5 \mathrm{y}^{2}\right]$ & $\mathrm{y}\left[\mathrm{R}^{2}-5 \mathrm{z}^{2}\right]$ \\
\hline $\mathrm{Q}_{\mathrm{xz}}$ & $\partial \mathrm{E}_{\mathrm{z}} / \partial \mathrm{x}$ & $\mathrm{z}\left[\mathrm{R}^{2}-5 \mathrm{x}^{2}\right]$ & $-5 \mathrm{xyz}$ & $\mathrm{x}\left[\mathrm{R}^{2}-5 \mathrm{z}^{2}\right]$ \\
\hline $\mathrm{Q}_{\mathrm{yz}}$ & $\partial \mathrm{E}_{\mathrm{z}} / \partial \mathrm{y}$ & $-5 \mathrm{xyz}$ & $\mathrm{z}\left[\mathrm{R}^{2}-5 \mathrm{y}^{2}\right]$ & $\mathrm{y}\left[\mathrm{R}^{2}-5 \mathrm{z}^{2}\right]$ \\
\hline
\end{tabular}

\section{Appendix B: Tables relating VSH multipolarity to atomic transitions.}

Below are a series of tables overviewing transitions for $\mathrm{d}-\mathrm{d}$, $\mathrm{f}-\mathrm{d}$ and $\mathrm{f}-\mathrm{f}$ transitions within different symmetry configurations. Note that, although we list where E0 photons would be placed, for completion of the tables, they do not exist in reality.

Table B.1: The spatial symmetry species and multipolarities of the possible d-d transitions of a transition metal ion in an octahedral $\left(\mathrm{O}_{\mathrm{h}}\right)$ environment.

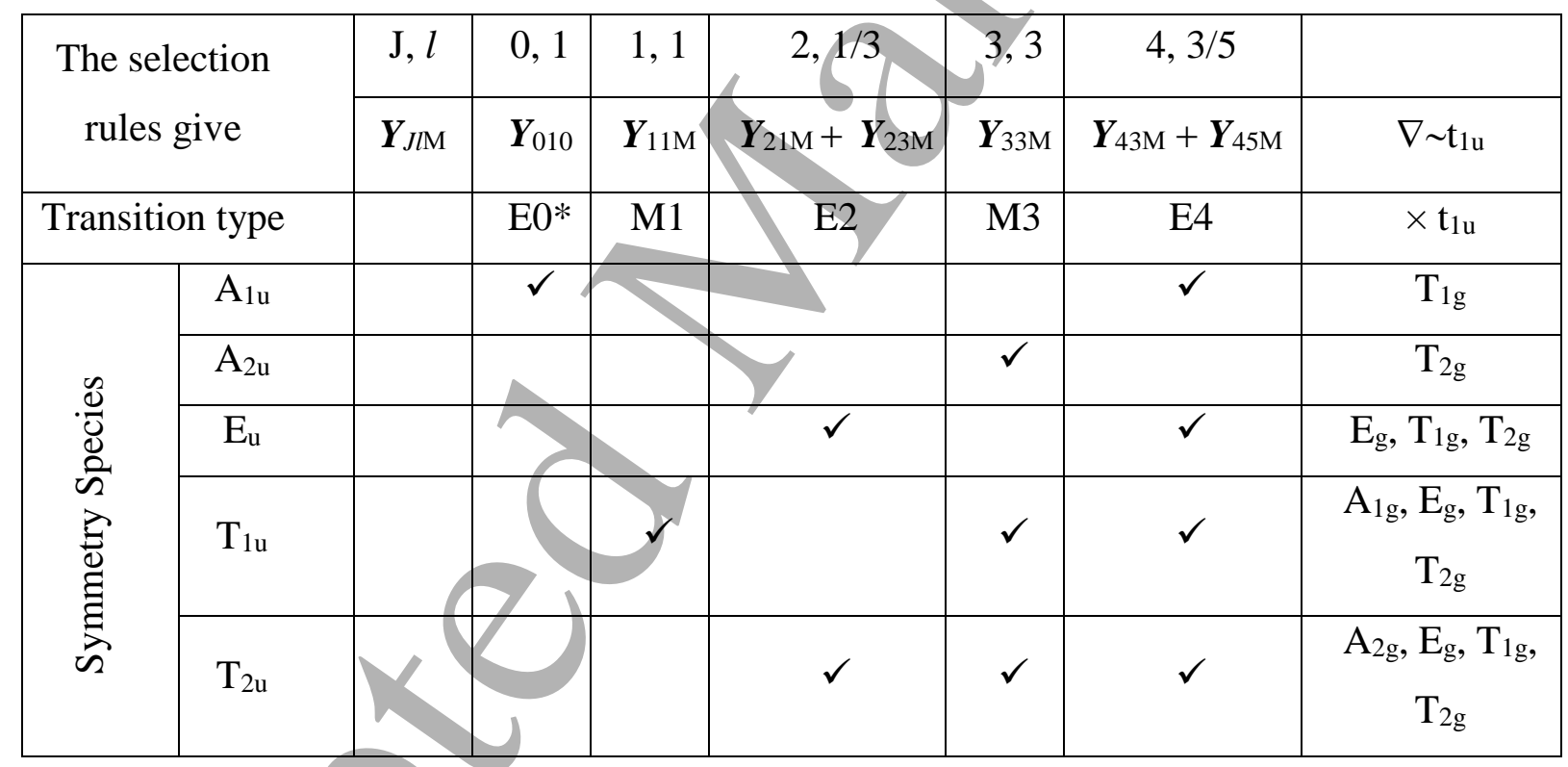

Table B.2: The spatial symmetry species and multipolarities of the possible d-d transitions of a transition metal ion in a tétrahedral $\left(\mathrm{T}_{\mathrm{d}}\right)$ environment.* $\mathrm{E} 0$ photons do not exist.

\begin{tabular}{|c|c|c|c|c|c|c|c|}
\hline $\begin{array}{c}\text { The selection } \\
\text { rules give }\end{array}$ & $\mathrm{J}, l$ & 0,1 & 1,1 & $2,1 / 3$ & 3,3 & $4,3 / 5$ & \\
\cline { 2 - 8 } & $\boldsymbol{Y}_{J l \mathrm{M}}$ & $\boldsymbol{Y}_{010}$ & $\boldsymbol{Y}_{11 \mathrm{M}}$ & $\boldsymbol{Y}_{21 \mathrm{M}}+\boldsymbol{Y}_{23 \mathrm{M}}$ & $\boldsymbol{Y}_{33 \mathrm{M}}$ & $\boldsymbol{Y}_{43 \mathrm{M}}+\boldsymbol{Y}_{45 \mathrm{M}}$ & $\nabla \sim \mathrm{t}_{2}$ \\
\hline Transition type & & $\mathrm{E} 0 *$ & $\mathrm{M} 1$ & $\mathrm{E} 2$ & $\mathrm{M} 3$ & $\mathrm{E} 4$ & $\times \mathrm{t}_{2}$ \\
\hline $\boldsymbol{\sim} \sim \mathrm{A}_{1}$ & & $\checkmark$ & & & $\checkmark$ & $\checkmark$ & $\mathrm{T}_{2}$ \\
\hline
\end{tabular}




\begin{tabular}{|c|c|c|c|c|c|c|c|c|}
\hline & $\mathrm{A}_{2}$ & & & & & & & \\
\hline & $\mathrm{E}$ & & & & $\checkmark$ & & $\checkmark$ & $\mathrm{T}_{1}, \mathrm{~T}_{2}$ \\
\hline & $\mathrm{T}_{1}$ & & & & & $\checkmark$ & $\checkmark$ & $\mathrm{A}_{2}, \hat{\mathrm{E}}_{\mathrm{N}} \mathrm{T}_{1}, \mathrm{~T}_{2}$ \\
\hline & $\mathrm{T}_{2}$ & & & $\checkmark$ & $\checkmark$ & $\checkmark$ & $\checkmark$ & $\mathrm{A}_{2}, \mathrm{E}, \mathrm{T}_{1}, \mathrm{~T}_{2}$ \\
\hline
\end{tabular}

Table B.3: The spatial symmetry species and multipolarities of the possible $d-d$ transitions of a transition metal ion in a square-planar $\left(\mathrm{D}_{4 \mathrm{~h}}\right)$ environment.* E0 photons do not exist.

\begin{tabular}{|c|c|c|c|c|c|c|c|c|}
\hline \multirow{2}{*}{\multicolumn{2}{|c|}{$\begin{array}{c}\text { The selection } \\
\text { rules give }\end{array}$}} & $\mathrm{J}, l$ & 0,1 & 1,1 & $2,1 / 3$ & 3,3 & & \\
\hline & & $\boldsymbol{Y}_{J l \mathrm{M}}$ & $\boldsymbol{Y}_{010}$ & $\boldsymbol{Y}_{11 \mathrm{M}}$ & $\boldsymbol{Y}_{21 \mathrm{M}}+\boldsymbol{Y}_{23 \mathrm{M}}$ & $\boldsymbol{Y}_{33 \mathrm{M}}$ & $\boldsymbol{Y}_{43 \mathrm{M}}+\boldsymbol{Y}_{45 \mathrm{M}}$ & $\nabla \sim\left(\mathrm{e}_{\mathrm{u}}+\mathrm{a}_{2 \mathrm{u}}\right)$ \\
\hline \multicolumn{2}{|c|}{$\begin{array}{l}\text { Transition } \\
\text { type }\end{array}$} & & $\mathrm{E} 0 *$ & M1 & E2 & & E4 & $\times\left(e_{u}+a_{2 u}\right)$ \\
\hline \multirow{5}{*}{ 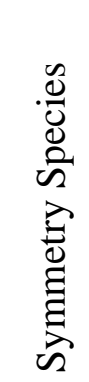 } & $\mathrm{A}_{1 \mathrm{u}}$ & & $\checkmark$ & & 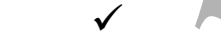 & & $\checkmark \checkmark$ & $E_{g}, A_{2 g}$ \\
\hline & $\mathrm{A}_{2 \mathrm{u}}$ & & & $\checkmark$ & & & $\checkmark$ & $E_{g}, A_{1 g}$ \\
\hline & $\mathrm{B}_{1 \mathrm{u}}$ & & & & & $\checkmark$ & $\checkmark$ & $E_{g}, B_{2 g}$ \\
\hline & $\mathrm{B}_{2 \mathrm{u}}$ & & & & & $\checkmark$ & $\checkmark$ & $E_{g}, B_{1 g}$ \\
\hline & $\mathrm{E}_{\mathrm{u}}$ & & & & & $\checkmark \checkmark$ & $\checkmark \checkmark$ & $A_{1 g}, A_{2 g}, B_{1 g}, B_{2 g}, E_{g}$ \\
\hline
\end{tabular}

Selection rules for $\mathrm{f}-\mathrm{d}$ transitions; $\boldsymbol{Y}_{J I \mathrm{M}} .1 \leq J \leq 5,|M| J, l$ must be even.

Table B.4: The spatial symmetry species and multipolarities of the possible f-d transitions of a transition metal ion in an octahedral $\left(\mathrm{O}_{\mathrm{h}}\right)$ environment.

\begin{tabular}{|c|c|c|c|c|c|c|c|}
\hline \multirow{2}{*}{$\begin{array}{c}\text { The selection } \\
\text { rules give }\end{array}$} & $\mathrm{J}, l$ & $1,0 / 2$ & 2,2 & $3,2 / 4$ & 4,4 & $5,4 / 6$ & \\
\hline & $\boldsymbol{Y}_{J I \mathrm{M}}$ & $\mathrm{M}+\boldsymbol{Y}_{12 \mathrm{M}}$ & $\boldsymbol{Y}_{22 \mathrm{M}}$ & $\boldsymbol{Y}_{32 \mathrm{M}}+\boldsymbol{Y}_{34 \mathrm{M}}$ & $\boldsymbol{Y}_{44 \mathrm{M}}$ & $Y_{54 \mathrm{M}}+\boldsymbol{Y}_{56 \mathrm{M}}$ & $\nabla \sim \mathrm{t}_{1 \mathrm{u}}$ \\
\hline Transition type & & E1 & $\mathrm{M} 2$ & E3 & M4 & E5 & $\times \mathrm{t}_{1 \mathrm{u}}$ \\
\hline$\geq \quad \mathrm{A}_{1 \mathrm{~g}}$ & & & & & $\checkmark$ & & $\mathrm{T}_{1 \mathrm{u}}$ \\
\hline$\stackrel{\mathscr{U}}{\Xi} \quad A_{2 g}$ & & & & $\checkmark$ & & & $\mathrm{T}_{2 \mathrm{u}}$ \\
\hline ते है & & & $\checkmark$ & & $\checkmark$ & $\checkmark$ & $\mathrm{T}_{1 \mathrm{u}}, \mathrm{T}_{2 \mathrm{u}}$ \\
\hline $\mathrm{T}_{1 \mathrm{~g}}$ & & $\checkmark$ & $\checkmark$ & $\checkmark$ & $\checkmark$ & $\checkmark \checkmark$ & $\mathrm{A}_{1 \mathrm{u}}, \mathrm{E}_{\mathrm{u}}, \mathrm{T}_{1 \mathrm{u}}, \mathrm{T}_{2 \mathrm{u}}$ \\
\hline
\end{tabular}




\begin{tabular}{|l|l|l|l|l|l|l|l|l|}
\hline & $\mathrm{T}_{2 \mathrm{~g}}$ & & & & $\checkmark$ & $\checkmark$ & $\checkmark$ & $\mathrm{A}_{2 \mathrm{u}}, \mathrm{E}_{\mathrm{u}}, \mathrm{T}_{1 \mathrm{u}}, \mathrm{T}_{2 \mathrm{u}}$ \\
\hline
\end{tabular}

Table B.5. The spatial symmetry species and multipolarities of the possible f-d transitions of a lanthanide ion in a $\mathrm{C}_{3 \mathrm{~h}}$ environment.

\begin{tabular}{|c|c|c|c|c|c|c|c|c|}
\hline \multirow{2}{*}{\multicolumn{2}{|c|}{$\begin{array}{c}\text { The selection } \\
\text { rules give }\end{array}$}} & $\mathrm{J}, l$ & $1,0 / 2$ & 2,2 & $3,2 / 4$ & 4,4 & $5,4 / 6$ & \\
\hline & & $\boldsymbol{Y}_{J l \mathrm{M}}$ & $\boldsymbol{Y}_{10 \mathrm{M}}+\boldsymbol{Y}_{12 \mathrm{M}}$ & $\boldsymbol{Y}_{22 \mathrm{M}}$ & $\boldsymbol{Y}_{32 \mathrm{M}}+\boldsymbol{Y}_{34 \mathrm{M}}$ & $\boldsymbol{Y}_{44 \mathrm{M}}$ & $\boldsymbol{Y}_{54 \mathrm{M}}+\boldsymbol{Y}_{56 \mathrm{M}}$ & $\nabla \sim\left(\mathrm{e}^{\prime}+\mathrm{a}^{\prime \prime}\right)$ \\
\hline \multicolumn{2}{|c|}{ Transition type } & & E1 & $\mathrm{M} 2$ & E3 & M4 & E5 & × (e' + a") \\
\hline \multirow{4}{*}{ 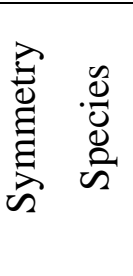 } & $\mathrm{A}^{\prime}$ & & & $\checkmark$ & $\checkmark \checkmark$ & $V$ & $\checkmark \checkmark$ & $E^{\prime}, A^{\prime \prime}$ \\
\hline & $E^{\prime}$ & & $\checkmark$ & $\checkmark$ & $\checkmark$ & & $\checkmark \checkmark$ & $\mathrm{E}^{\prime}, 2 \mathrm{~A}, \mathrm{E}^{\prime \prime}$ \\
\hline & $A^{\prime \prime}$ & & $\checkmark$ & & $\checkmark$ & $\sqrt{ }$ & $\checkmark$ & $E^{\prime \prime}, A^{\prime}$ \\
\hline & $E^{\prime \prime}$ & & & $\checkmark$ & $\sqrt{ }$ & & $\checkmark \checkmark$ & E", 2A"', E' \\
\hline
\end{tabular}

Selection rules for $\mathrm{f}-\mathrm{f}$ transitions; $\boldsymbol{Y}_{J I \mathrm{M} .} 0 \leq J \leq 6, M \leq J, l$ must be odd.

Table B.6: The spatial symmetry species and multipolarities of the possible f-f transitions of a lanthanide ion in a $\mathrm{C}_{3 \mathrm{~h}}$ environment.* $\mathrm{E} 0$ photons do not exist.

\begin{tabular}{|c|c|c|c|c|c|c|c|c|c|c|}
\hline \multirow{2}{*}{\multicolumn{2}{|c|}{$\begin{array}{c}\text { The selection } \\
\text { rules give }\end{array}$}} & $\mathrm{J}, l$ & 0,1 & 1,1 & $2,1 / 3$ & 3,3 & $4,3 / 5$ & 5,5 & $6,5 / 7$ & \\
\hline & & $\boldsymbol{Y}_{J l \mathrm{M}}$ & $\boldsymbol{Y}_{010}$ & & $\begin{array}{r}Y_{21 \mathrm{M}}+ \\
\boldsymbol{Y}_{23 \mathrm{M}}\end{array}$ & $\boldsymbol{Y}_{33 \mathrm{M}}$ & $\begin{array}{r}\boldsymbol{Y}_{43 \mathrm{M}}+ \\
\boldsymbol{Y}_{45 \mathrm{M}}\end{array}$ & $\boldsymbol{Y}_{55 \mathrm{M}}$ & $\begin{array}{c}\boldsymbol{Y}_{65 \mathrm{M}}+ \\
\boldsymbol{Y}_{67 \mathrm{M}}\end{array}$ & $\nabla \sim\left(\mathrm{e}^{\prime}+\mathrm{a}^{\prime \prime}\right)$ \\
\hline \multicolumn{2}{|c|}{ Transition type } & & & M1 & E2 & M3 & E4 & M5 & E6 & $\times\left(\mathrm{e}^{\prime}+\mathrm{a}^{\prime \prime}\right)$ \\
\hline \multirow{4}{*}{ 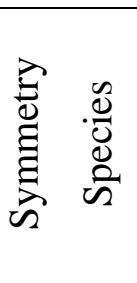 } & $\mathrm{A}^{\prime}$ & & & & $\checkmark$ & $\checkmark \checkmark$ & $\checkmark$ & $\checkmark \checkmark$ & $\checkmark \checkmark \checkmark$ & $E^{\prime}, A^{\prime \prime}$ \\
\hline & $\mathrm{E}^{\prime}$ & & & $\checkmark$ & $\checkmark$ & $\checkmark$ & $\checkmark \checkmark$ & $\checkmark \checkmark$ & $\checkmark \checkmark$ & $\mathrm{E}^{\prime}, 2 \mathrm{~A}^{\prime}, \mathrm{E}^{\prime \prime}$ \\
\hline & $\mathrm{A}^{\prime \prime}$ & & & $\checkmark$ & & $\checkmark$ & $\checkmark \checkmark$ & $\checkmark$ & $\checkmark \checkmark$ & $E^{\prime \prime}, A^{\prime}$ \\
\hline & $\mathrm{E}$ & & & & $\checkmark$ & $\checkmark$ & $\checkmark$ & $\checkmark \checkmark$ & $\checkmark \checkmark$ & $\mathrm{E}^{\prime \prime}, 2 \mathrm{~A}^{\prime \prime}, \mathrm{E}^{\prime}$ \\
\hline
\end{tabular}

\section{References}

1. Rubinsztein-Dunlop, H., et al., J.Opt., 2017. 19(1): p. 013001.

2. Tsang, M and Psaltis D 2007 Opt. Express 1511959

3. Adams W, Sadatgol M and Güney D Ö 2016 AIP Advances 6100701

4. Bazylewski P, Ezugwu S and Fanchini G 2017 Appl. Sci. 7973 
5. Padgett M J and Boyd R W 2017 Phil. Trans. R. Soc. A 375: 20160233

6. Ragy S and Adesso G 2012 Scientific Reports 2651

7. Ladd T D, Jelezko F, Laflamme R, Nakamura Y, Monroe C and O’Brien J L 2010 Nature 46445

8. Nielsen M A and Chuang I Quantum Computation and Quantum Information (Cambridge University Press, Cambridge, 2010)

9. Duan L M, Lukin M D, Cirac J I, Zoller P 2001 Nature 414413

10. Liao S-K, et al. 2017 Nat. Photonics 11509

11. Dirac P M A 1926 Proc. Roy. Soc. 112661

12. Dirac P. M. A 1927 Proc. Roy. Soc. 114243710

13. Jordan P and Pauli W 1928 Zeit. für Phys. 47151

14. Schwinger J 1948 Phys. Rev. 741439 - 61

15. Feynman R. P. 1949 Phys. Rev. 76769 - 89

16. Craig D. P. and Thirunamachandran T. Molecular Quantum Electrodynamics (Dover, New York, 1998)

17. Conway A W 1932 Proc. Roy. Irish Acad. 418

18. Heitler W 1936 Proc. Camb. Phil. Soc. 37112

19. Franz W 1950 Zeit. für Phys. 127363

20. Brink D M and Satchler G R 1968 Angular Momentum (Oxford: Oxford University Press)

21. Blatt J M and Weisskopf V F 1991 Theoretical Nuclear Physics (New York: Dover)

22. Goldhaber M and Sunyar A W Beta and Gamma-Ray Spectroscopy (Ed. K. Siegbahn, North Holland Publishing Company, Amsterdam 1955).

23. Andrews D.L., Structured Light and its Applications: An Introduction to Phase-Structured Beams and Nanoscale Optical Forces. 2008, Amsterdam, Boston: Academic.

24. Yao A.M. and Padgett M J, Adv. Opt. Photon., 2011. 3(2): p. 161-204.

25. Watson G N 1944 A Treatise on the Theory of Bessel Functions (Cambridge: Cambridge University Press, $2^{\text {nd }}$ Edtn.)

26. Abramowitz M and Segun IA 1965Handbook of Mathematical Functions (New York: Dover)

27. Rose M E 1995 Elementary Theory of Angular Momentum (New York: Dover)

28. Power E A, Introductory Quantum Electrodynamics (Longmans, London, 1964)

29. Loudon R, The Quantum Theory of Light, 2nd Edition (Clarendon Press, Oxford, 1983)

30. Cohen-Tannoudji C, Dupont-Roc J and Grynberg G, Photons and Atoms: Introduction to Quantum Electrodynamics (John Wiley \& Sons, Inc., New York, 1989)

31. Woolley R G, Handbook of Molecular Physics and Quantum Chemistry Ed. S. Wilson, 1, Part 7, Ch. 38 (2003).

32. Schiff L I, Quantum Mechanics (McGraw-Hill, New York, 1949)

33. Powell D A, Lapine M, Gorkunov M V, Shadrivov I V and Kivshar 2010 Phys. Rev. B 82155128

34. Cui T J, Smith D. R., Ruopeng, L, Metamaterials: Theory, Design, and Applications (Springer, New York, 2010)

35. Power E A and Zienau S 1959 Phil. Trans. R. Soc. A 251, 427

36. Atkins P W and Woolley R G 1970 Proc. R. Soc. A 319, 549. 
37. Woolley R G 1971 Proc. R. Soc. A 321, 557.

38. Andrews D L, Bradshaw D S 2004 Eur. J. Phys. 25 845-858.

39. Lock M E P, Andrews D L, Jones G A, 2014 J. Chem. Phys. 140044103.

40. Frost J E, Jones G A 2014 New J. Phys. 16113067.

41. Brink D M and Satchler G R 1968 Angular Momentum (Oxford: Oxford University Press)

42. Edmonds A R 1957 Angular Momentum in Quantum Mechanics (Princeton, N.J: Princeton University press)

43. Blatt J M and Weisskopf V F 1991 Theoretical Nuclear Physics (New York: Dover)

44. Grinter R 2014 J. Phys. B: At. Mol. Opt. Phys. 47075004

45. Cowan R D 1981 The Theory of Atomic Structure and Spectra (Berkley: University of California Press)

46. Rotenberg M, Bivins R, Metropolis N and Wooten J K 1959 The 3j and 6j Symbols (Cambridge: MIT Technology Press)

47. Grant I P 1974 J. Phys. B:Atom. Mol. Phys. 7 1458-1475.

48. Glushkov A V, Malinovskaya S V, Khetselius O. Yu., Loboda, A V, Sukharev D E, Lovett, L 2009 J. Quant. Chem. 109 1717-1727.

49. Grinter R 2008 J. Phys. B: At. Mol. Opt. Phys. 41095001

50. Salam A, Molecular Quantum Electrodynamics (John Wiley \& Sons, Inc., Hoboken, 2010)

51. Rose M E 1955 Multipole Fields (New York: Wiley)

52. Condon E U and Shortley G H 1935 The Theory of Atomic Spectra (Cambridge: Cambridge University Press)

53. Huff L D and Houston W V 1930 Phys. Rev. 36842

54. Bartholemew G A 1960 in Nuclear Spectroscopy part A, ed. Ajzenberg-Selove F (New York: Academic Press)

55. Condon E U and Shortley G/H 1935 The Theory of Atomic Spectra (Cambridge: Cambridge University Press)

56. Andrews D L Phys. Rev., A81, 033825, 2010

57. Rubinowicz A 1929 Zeit. für Phys. 53 267; 1930 Zeit. für Phys. 61338

58. Huff L D and Houston W V 1930 Phys. Rev. 36842

59. Born M and Wolf E1959 Principles of Optics (Oxford: Pergamon); $6^{\text {th }}$ edn (1980 Cambridge: Cambridge University Press) pp 28-30 (Note that some earlier editions cite a relation between the sign of $\operatorname{Im}\left\{\Phi_{J, l, M} / \Theta_{J, l, M}\right\}$ and chirality which applies to the definition of chirality used in nuclear physics, rather than that used in optics. This is changed in later editions.

60. Rice E M, Bradshaw D S, Saadi K and Andrews D L 2012 Eur. J. Phys. 33345

61. Jackson J D 1999 Classical Electrodynamics (New York: Wiley)

62. Avery J S 1966 Proc. Phys. Soc. 88, 1

63. Power E A and Thirunamachandran T 1983 Phys. Rev. A28 2649

64. Power E A and Thirunamachandran T 1983 Phys. Rev. A28 2663

65. Power E A and Thirunamachandran T 1983 Phys. Rev. A28 2671

66. Andrews D L and Sherborne B 1987 J. Chem. Phys. 86, 4011 
67. Andrews D L 1989 Chem. Phys. 135, 195

68. Andrews D L and Juzeliunas G 1992, J. Chem. Phys. 96, 6606

69. Scholes G D and Andrews D L 1997 J. Chem. Phys. 107, 5374

70. Andrews D L and Demidov A A Resonance Energy Transfer (Wiley, New York, 1999)

71. Daniels G J, Jenkins R D, Bradshaw D S and Andrews D L, 2003 J. Chem. Phys. 119, 264

72. Stout B, Devilez A, Rolly B and Bonod N 2011 J. Opt. Soc. Am. B 281213

73. Grinter R G and Jones G A 2016 J. Chem. Phys., 145, 074107

74. Craig D P and Hobbins J. Chem. Soc. 539, 1955

75. Craig D P J. Chem. Soc. 2302, 1955

76. Ballhausen C J 1962 Introduction to Ligand Field Theory (New York: McGraw-Hill)

77. Dieke G. H. 1968 Spectra and Energy Levels of Rare Earth Ions in Crystals (New York: Wiley Interscience)

78. C. K. Jørgensen, Absorption Spectra and Chemical Bonding in Complexes (Cambridge: Cambridge University Press, 1962)

79. Griffith J S 1961 The Theory of Transition Metal Ions (Cambridge: Cambridge University Press)

80. Rodrigues M O, Dutra, J D L, Nunes L A O, De Sá G F, De Azevedo W M, Silva P, Paz F A A, Freire R O and Júnior S A 2012 J. Phys. Chem. C 11619951

81. Sin C, Aidilibike T, Qin W and Yu C-J 2018 J. Lumin. 19472.

82. Piepho S B and Schatz P N 1983 Group Theory in Spectroscopy with Applications to Magnetic Circular Dichroism (New York: Wiley)

83. Salthouse J A and Ware MJ 1972 Point Group Character Tables and Related Data (Cambridge: Cambridge University Press)

84. Cowan R D 1981 The Theory of Atomic Structure and Spectra (Berkley: University of California Press)

85. Griffith J S 1962 The Irreducible Tensor Method for Molecular Symmetry Groups (Englewood Cliffs, N.J.:Prentice-Hall)

86. Koster G F, Dimmock J O, Wheeler RG and Statz H 1963 Properties of the Thirty-Two Point Groups (Cambridge MA: MIT Press)

87. Wigner EP 1959 Group Theory (New York: Academic Press)

88. Racah G 1942 Phys. Rev. 62 438-462

89. Racah G 1943 Phys. Rev. $633678-382$

90. Racah G 1949 Phys. Rev. 76 1352-1365

91. Butler PH 1975 Phil. Trans. R. Soc. London 277, 545

92. Butler PH 1981 Point Group Symmetry Applications (New York: Plenum Press)

93. Piepho S B and Schatz P N 1983 Group Theory in Spectroscopy with Applications to Magnetic Circular Dichroism (New York: Wiley)

94. Mason S F1963 Optical Rotatory Power, Quart. Rev. Chem. Soc., 1720

95. Grinter R and Jones G A, 2018 Opt. Lett. 43, 367 